\title{
Coating Flows of Non-Newtonian Fluids: Weakly and Strongly Elastic Limits
}

\author{
J. Ashmore ${ }^{(1, a)}$, A.Q. Shen ${ }^{(1, b)}$, H.P. Kavehpour ${ }^{(2, c)}$, H.A. Stone ${ }^{(1)} \&$ \\ G.H. McKinley ${ }^{(2)}$ \\ 1: Division of Engineering and Applied Sciences, Harvard University, \\ Cambridge, MA 02138 \\ 2: Hatsopoulos Microfluidics Laboratory, Department of Mechanical \\ Engineering, Massachusetts Institute of Technology, Cambridge, MA 02139 \\ (a): Current address: TIAX LLC, 15 Acorn Park, Cambridge, MA 02140 \\ (b): Current address: Department of Energy, Environmental \& Chemical \\ Engineering, Washington University in St Louis, St Louis, MO 63130 \\ (c): Current address: Department of Mechanical and Aerospace Engineering, \\ University of California, Los Angeles, CA 90095
}

10th February 2007

\begin{abstract}
.
We present an asymptotic analysis of the thickness of the liquid film that coats a smooth solid substrate when it is withdrawn from a bath of non-Newtonian fluid, and compare our results with experimental measurements. The film thickness is, to a good approximation, uniform above the point where the film is withdrawn from the fluid bath, and depends on the rotation rate, the fluid properties and the substrate geometry. Theoretical predictions of the film thickness for a number of different substrate geometries (an inclined plate, roller and fiber) are presented, and are compared with experimental measurements in a single roller geometry. Results are obtained for two different limits of the Criminale-Ericksen-Filbey constitutive equation in which the fluid rheology is either weakly elastic and dominated by shear-thinning, or strongly elastic and dominated by elastic stresses. A lubrication analysis yields a thin-film equation which characterizes the film thickness as a function of spatial position. The rheological properties of the test fluids are measured independently using steady and oscillatory shearing deformations. The viscometric parameters are then used, in conjunction with the governing thin-film equation, which is solved using matched asymptotics, to give a quantitative prediction of the thickness of the fluid coating. The onset of an instability which causes the film thickness to vary with axial position along the roller is also observed experimentally.
\end{abstract}

\section{Introduction}

Many industrial processes require the deposition of a thin film of liquid onto a substrate, and the fluids utilized often have non-Newtonian properties. It is usually desired to be able to control the film thickness. One way to achieve control is to meter the film using a blade; another is to allow it to be pulled out without any external interference, in which case the thickness is controlled by the substrate velocity, fluid

(C) 2007 Kluwer Academic Publishers. Printed in the Netherlands. 
properties and the geometric configuration (Ruschak, 1985). Studies have covered a number of different substrate geometries, a variety of constitutive equations and both low and high capillary number (dimensionless withdrawal speed) processes. Our focus here is on viscoelastic effects on single roll coating at low dimensionless speeds, although the analytical results we describe are general and cover a variety of substrate geometries. As we show below, the appropriate dimensionless parameter characterizing low speeds will vary in the two different rheological limits we examine.

Landau and Levich (1942) presented a seminal theory that characterized the thickness of the film of Newtonian fluid which is deposited on a plate that is withdrawn vertically from a bath at constant velocity $U$; Derjaguin (1943) subsequently extended this work to the coating of a cylindrical fiber. Finally, Bretherton (1961) showed that a similar analysis could be used to characterize the thickness of the uniform film that (when wetting properties of the liquid on the surface are favorable) surrounds a bubble as it advances into a cylindrical tube of Newtonian liquid. In each case, the analytical method was based on matched asymptotics and the first term in an asymptotic expansion in powers of $\mathcal{C}_{N}^{1 / 3}$ was derived, where $\mathcal{C}_{N}=\eta U / \sigma$ is the capillary number for a Newtonian fluid, $\eta$ is the viscosity and $\sigma$ is the surface tension. Wilson (1981) later published a detailed asymptotic analysis for a plate withdrawn at an angle $\theta$ from a bath of Newtonian fluid, and noted that this theory also applies to a rotating cylindrical roller lying with its axis horizontal so that it is partially submerged in the bath.

Many papers, both theoretical and experimental, have followed and several review articles are available (Kistler and Scriven, 1983; Quéré, 1999; Ruschak, 1985). Several studies have adapted the asymptotic analysis to account for non-Newtonian effects, which are the primary focus in the present work. Here we review some results from published studies on Newtonian and non-Newtonian coating flows, focusing mainly on the plate and roller geometries. It is worth noting that when the radius of the roller is large compared to the capillary length $\ell_{c}=\sqrt{\sigma / \rho g}$ where $\rho$ is the fluid density and $g$ is the gravitational constant, then the effect of the curvature of the cylinder is very weak and results in only small perturbations to the uniform film thickness on the roller. To leading order the thickness of a film coating a roller which meets the fluid bath at a local angle $\alpha$ is the same as that coating a plate inclined at an angle $\alpha$. A summary of the literature, detailing the geometry and the constitutive equation used, is presented in Table I. This tabular summary is not intended to be comprehensive but to focus primarily on key developments for the plate and roller geometries. Some details are discussed below. 
Table I. Partial summary of relevant literature on coating with a Newtonian and non-Newtonian liquid. MA indicates papers in which matched asymptotic methods were used. Analysis based on matched asymptotics is restricted to low nondimensional speeds; a variety of other methods (some adhoc) are used at high nondimensional speeds.

\begin{tabular}{|c|c|c|c|}
\hline Authors & $\begin{array}{l}\mathrm{T} \text { : theory, } \\
\mathrm{N} \text { : numerical, } \\
\mathrm{E} \text { : experiment }\end{array}$ & Geometry & $\begin{array}{l}\text { Constitutive } \\
\text { equation }\end{array}$ \\
\hline Landau and Levich (1942) & $\mathrm{T}(\mathrm{MA})$ & flat plate & Newtonian \\
\hline Derjaguin (1943) & $\mathrm{T}(\mathrm{MA})$ & fiber & Newtonian \\
\hline Bretherton (1961) & $\mathrm{T}(\mathrm{MA})$ & bubble in tube & Newtonian \\
\hline Gutfinger and Tallmadge (1965) & $\mathrm{T}(\mathrm{MA}), \mathrm{E}$ & flat plate & power-law \\
\hline Tallmadge (1966) & $\mathrm{T}$ & flat plate & Ellis \\
\hline Spiers et al. (1974) & $\mathrm{T}, \mathrm{E}$ & flat plate & Newtonian \\
\hline Spiers et al. (1975) & $\mathrm{T}, \mathrm{E}$ & flat plate & $\begin{array}{l}\text { Bingham, Ellis } \\
\text { \& power-law }\end{array}$ \\
\hline Middleman (1978) & $\mathrm{E}$ & roller & (no theory) \\
\hline \multicolumn{4}{|l|}{ Tharmalingham and } \\
\hline Wilkinson (1978) & $\mathrm{T}, \mathrm{N}, \mathrm{E}$ & roller & Newtonian \\
\hline Cerro and Scriven (1980) & $\mathrm{N}$ & flat plate & Newtonian \\
\hline Wilson (1981) & $\mathrm{T}(\mathrm{MA})$ & inclined plate & Newtonian \\
\hline Campanella et al. (1982b) & $\mathrm{T}, \mathrm{E}$ & roller & power-law \\
\hline Campanella and Cerro (1982a) & $\mathrm{T}, \mathrm{N}, \mathrm{E}$ & roller & Newtonian \\
\hline Ro and Homsy (1995) & $\begin{array}{l}\mathrm{T}(\mathrm{MA} \& \\
\text { perturbation } \\
\text { analysis) }\end{array}$ & $\begin{array}{l}\text { flat plate, } \\
\text { fiber, } \\
\text { bubble in tube }\end{array}$ & Oldroyd-B \\
\hline de Ryck and Quéré (1998) & $\mathrm{T}(\mathrm{MA}), \mathrm{E}$ & fiber & viscoelastic \\
\hline Kizito et al. (1999) & $\mathrm{E}$ & roller & Newtonian \\
\hline
\end{tabular}

A number of studies have focused on coating at slow nondimensional speeds. For liquids described by the Newtonian or generalized Newtonian constitutive equation, slow speeds correspond to small capillary numbers $\mathcal{C}=\eta(\dot{\gamma}) U / \sigma$, where in general the viscosity $\eta(\dot{\gamma})$ may depend on the shear rate $\dot{\gamma}$. For the viscoelastic fluid studied by de Ryck and Quéré (1998) and analysed in more detail in this paper, slow speeds are quantified by a different nondimensional parameter that incorporates the magnitude of elastic stresses. This dimensionless measure of elasticity will be derived in section 2.5. In the low capillary number limit, a theoretical prediction may be derived using matched asymptotic methods. Many researchers have considered generalized Newtonian models; for example, Tallmadge (1966) analyzed the problem of coating a flat 
plate using the Ellis constitutive model. Comparison between theory and experiments has met with various degrees of success. Gutfinger and Tallmadge (1965) developed theory and performed experiments for coating a plate with a power-law fluid, and found qualitative but not quantitative agreement. Spiers et al. (1975) compared theory for the Bingham, Ellis and power-law models with experiments on coating a plate with numerous fluids. As these latter authors commented, only a small number of their experimental results were in good agreement with their theoretical predictions.

The reasons for the discrepancies noted above can be traced to the wide variety of non-Newtonian responses that can occur in complex fluids. These include rate-dependence of the material functions, a natural relaxation time providing memory of the previous deformation history, and elastic normal stress differences. It is difficult to parametrize such effects using a single dimensionless parameter such as a capillary number and care must be taken to perform asymptotic analyses and comparisons with experimental data in appropriate limits. Only a few studies have explicitly considered viscoelastic effects. Ro and Homsy (1995) performed a perturbation analysis based on the Oldroyd-B model which incorporated the effects of small normal stress differences to determine the thickness of the film left behind when a bubble advances in a Hele-Shaw cell, as well as the thickness of the film deposited on a flat plate or large roller withdrawn from a liquid. In the fiber coating geometry, de Ryck and Quéré (1998) compared the prediction of a viscoelastic constitutive model valid for steady viscometric flows with experimental results for concentrated polymer solutions coating a fiber and found good agreement. The work in this paper is based on a similar constitutive equation to that used by de Ryck and Quéré (1998), and in section 2.5 we present a formal matched asymptotic analysis of the limit that they studied, generalized to cover a number of substrate geometries.

The geometry and notation used in this paper are indicated in Figure 1 . The film thickness $h\left(x_{1}\right)$ reaches a constant value $h_{\infty}$ away from the region of meniscus curvature where the film is withdrawn from the fluid bath. The constant film thickness $h_{\infty}$ may depend on the substrate velocity $U$, fluid density $\rho$, viscosity $\eta(\dot{\gamma})$, which may vary with the shear rate $\dot{\gamma}$, first normal stress coefficient $\Psi_{1}(\dot{\gamma})$, surface tension $\sigma$, gravitational acceleration $g$, a substrate length-scale $L$ and the angle of inclination $\alpha$. In this paper we present theoretical predictions of the film thickness based on the Criminale-Ericksen-Filbey (CEF) constitutive equation in "weakly" and "strongly" elastic limits. (A mathematical definition of weakly and strongly elastic limits will be given in section 2.3.) The CEF model (Bird et al., 1987) is a nonlinear constitutive 


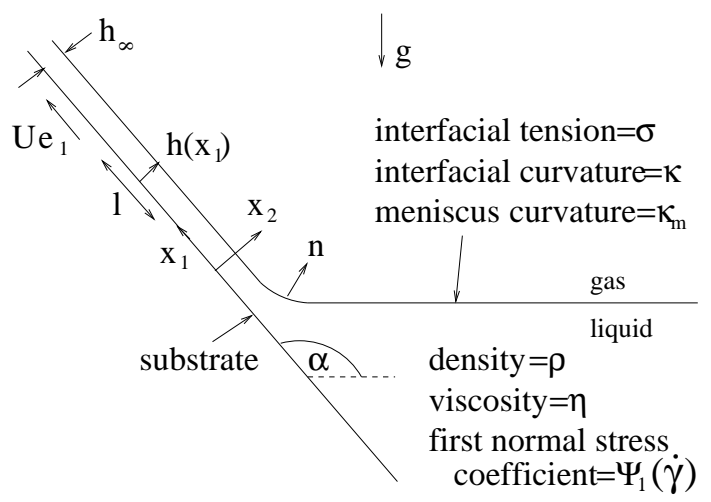

Figure 1. Withdrawal of a substrate from a bath, which results in a thin film of fluid being deposited onto the substrate. Material parameters and parameters that characterize the interface shape are indicated.

equation applicable to weak or strong viscometric flows which are dominated by shearing deformation. The total stress (including the isotropic pressure term) $\boldsymbol{\tau}$ is represented by:

$$
\boldsymbol{\tau}=-p \mathbf{I}+\eta(\dot{\gamma}) \dot{\gamma}-\frac{1}{2} \Psi_{1}(\dot{\gamma}) \dot{\gamma}_{(1)}+\Psi_{2}(\dot{\gamma}) \dot{\gamma} \cdot \dot{\gamma}
$$

where all variables are dimensional and $p$ is the pressure. The viscosity, $\eta(\dot{\gamma})$, and the first and second normal stress coefficients, $\Psi_{1}(\dot{\gamma})$ and $\Psi_{2}(\dot{\gamma})$ respectively, are arbitrary specified functions of the shear rate $\dot{\gamma}=\sqrt{\dot{\gamma} \cdot \dot{\gamma} / 2}$ which can be chosen to fit the measured experimental data, and the rate of strain tensor $\dot{\gamma}=\nabla \mathbf{u}+(\nabla \mathbf{u})^{T}$. Finally, the tensor $\dot{\gamma}_{(1)}$ is the first upper-convected derivative of the rate of strain tensor and is defined by

$$
\dot{\gamma}_{(1)}=\frac{\partial \dot{\gamma}}{\partial t}+\mathbf{u} \cdot \nabla \dot{\gamma}-\left(\dot{\gamma} \cdot \nabla \mathbf{u}+(\nabla \mathbf{u})^{T} \cdot \dot{\gamma}\right) .
$$

The power-law like forms of the viscosity and first normal stress difference that we choose in the present work, explained in section 2.2, are such that in the weakly elastic limit the CEF constitutive equation reduces to the Newtonian or generalized Newtonian fluid constitutive equation, depending on the value of a single nondimensional parameter in the general functional form chosen for the viscosity $\eta(\dot{\gamma})$.

The present work generalizes previous analysis of power-law fluids in coating flows in plate and roller geometries (see e.g. Gutfinger and Tallmadge, 1965) and study of viscoelastic flows in the fiber geometry (de Ryck and Quéré, 1998). The only previous theoretical study of normal stress effects is by Ro and Homsy (1995), who used matched asymptotic methods and perturbation analysis to explore the effects of 
relatively weak normal stresses on coating flows using the Oldroyd-B constitutive equation. To the best of our knowledge, we present the first rigorous matched asymptotic analysis of coating flows based on the CEF constitutive equation. The thickness of the fluid film that coats the sides and top of a horizontal rotating roller that is partially immersed in a bath of fluid is also measured experimentally over a range of rotation rates and depths of immersion.

In section 2 we outline the model formulation, based on the CEF constitutive equation, derive the thin-film equation governing the film thickness and characterize predictions for the film thickness in the weakly- and strongly-elastic limits. The experimental setup is described in section 3. Experimental results for two different non-Newtonian fluids, one which is weakly viscoelastic but exhibits a strongly shearthinning viscosity, and another fluid with strong viscoelastic characteristics, are presented in section 4 . Using the rheological data to determine the viscometric parameters in the model we compare experimental results to the theoretical predictions. We discuss our results in section 5 and present conclusions in section 6 .

\section{Model}

We consider a substrate withdrawn from a fluid bath at a sufficiently high speed that a continuous thin film of fluid is entrained on the substrate, as shown in the schematic diagram in Figure 1. The analysis encompasses different substrate geometries, which may be either planar, or a roller with curvature in the direction of motion, or a cylindrical fiber. Variations in film thickness are negligible above the meniscus which rises a small distance above the pool for all the substrate geometries discussed here. The analysis also applies to the thickness of the film that coats the walls behind the nose of a bubble advancing in a cylindrical capillary tube provided the fluid wets the tube walls. We use the lubrication approximation to the momentum equations to derive a nonlinear third-order differential equation that describes the film thickness, and use matched asymptotic analysis and numerical solutions to the thin-film equation to predict the constant thickness $h_{\infty}$ that the film attains away from the meniscus region. The theoretical predictions for the film thickness are only compared to experiments for the single roller geometry, but we also indicate the theoretical predictions for plate and fiber geometries.

The analysis we present focuses on two-dimensional variations, and the coordinates $x_{1}, x_{2}$ are indicated in Figure 1. The origin for $x_{1}$ is the point where the static meniscus would meet the substrate at 
zero contact angle. Also, $\mathbf{e}_{1}$ and $\mathbf{e}_{2}$ are unit vectors in the $x_{1^{-}}$and $x_{2}$-directions respectively and the substrate is withdrawn at velocity $U \mathbf{e}_{1}$. We denote the steady velocity field by $\mathbf{u}\left(x_{1}, x_{2}\right)$, the pressure by $p\left(x_{1}, x_{2}\right)$ and the stress tensor by $\boldsymbol{\tau}$. The fluid density is $\rho, \mathbf{g}$ is the gravitational force per unit mass, $\sigma$ is the surface tension and the interface $x_{2}=h\left(x_{1}\right)$ has unit normal $\mathbf{n}$ and curvature $\kappa$. The expression for the curvature is dependent on the geometry of the substrate: $\kappa=$ $h^{\prime \prime} /\left(1+h^{\prime 2}\right)^{3 / 2}$ for a plane, where ' denotes differentiation with respect to $x_{1}, \kappa=\left(h / R^{2}+h^{\prime \prime}\right) /\left(1+h^{\prime 2}\right)^{3 / 2}$ for a roller of radius $R$ and $\kappa=$ $\left((R+h)^{-1}+h^{\prime \prime}\right) /\left(1+h^{\prime 2}\right)^{3 / 2}$ for a fiber of radius $R$. For generality, we denote the curvature by the symbol $\kappa$.

In addition, $\kappa_{m}$ is the curvature of the static meniscus and also depends on the substrate geometry. For example, for a flat plate inclined at angle $\alpha$ to the horizontal, as indicated in Figure $1, \kappa_{m}=$ $\sqrt{2(1+\cos \alpha)} / \ell_{c}$ where $\ell_{c}=\sqrt{\sigma / \rho g}$ is the capillary length. Note that the appropriate value of $\alpha$ is different for the underside or upper surface of the plate: when calculating the film thickness coating the underside, $0<\alpha<\pi / 2$, whereas when calculating the thickness on the upper side, $\pi / 2<\alpha<\pi$. For a roller with radius $R \gg \ell_{c}$, the curvature is also $\kappa_{m}=\sqrt{2(1+\cos \alpha)} / \ell_{c}$, where $\alpha$ is the angle subtended locally at the point where the meniscus forms on the roller. Finally, for a fiber with radius $R \ll \ell_{c}$ withdrawn vertically, $\kappa_{m}=1 / R$. Again, we use the notation $\kappa_{m}$ to retain generality.

The dimensional time-independent continuity and Cauchy momentum equations for incompressible flows are

$$
\nabla \cdot \mathbf{u}=0, \quad \rho \mathbf{u} \cdot \nabla \mathbf{u}=\nabla \cdot \boldsymbol{\tau}+\rho \mathbf{g},
$$

which are to be solved subject to the boundary conditions

$$
\mathbf{u}\left(x_{1}, 0\right)=U \mathbf{e}_{1}, \quad \mathbf{n} \cdot \boldsymbol{\tau}=\left(-p_{0}+\sigma \kappa\right) \mathbf{n} \quad \text { on } x_{2}=h\left(x_{1}\right),
$$

where $p_{0}$ is atmospheric pressure and $U$ is the substrate velocity; in the case of a roller of radius $R$ rotating at a rate $\Omega, U=\Omega R$. In section 2.1 we introduce the nondimensionalization used in the subsequent analysis and the dimensionless parameters that appear in the problem; the Criminale-Ericksen-Filbey constitutive equation is presented subsequently in section 2.2 , and we use this to derive the thin-film equation that forms the basis of our analysis in section 2.3.

\subsection{NONDIMENSIONALIZATION}

We nondimensionalize the equations (3) and boundary conditions (4) to obtain the lubrication creeping flow equations, which are commonly used in studies of coating problems (Bretherton, 1961; Derjaguin, 
1943; Landau and Levich, 1942; Wilson, 1981). In order that a lubrication analysis may be applied, different characteristic length-scales are introduced in the $x_{1}$ - and $x_{2}$-directions. The length-scale in the $x_{1}$-direction parallel to the substrate is denoted by an unknown $\ell$; physically it corresponds to the length-scale over which free-surface curvature variations are significant. It is important to note that $\ell$ will scale differently in analysis of the weakly and strong elastic limits of the $\mathrm{CEF}$ equation. The length-scale in the $x_{2}$-direction perpendicular to the substrate is characterized by the film thickness far away from the pool, $h_{\infty}$. We consider the lubrication limit $h_{\infty} / \ell \ll 1$.

Nondimensional variables, indicated by an overbar, are defined as follows

$$
\begin{aligned}
& \bar{u}_{1}=\frac{u_{1}}{U}, \quad \bar{u}_{2}=\frac{\ell u_{2}}{h_{\infty} U}, \quad \bar{x}_{1}=\frac{x_{1}}{\ell}, \quad \bar{x}_{2}=\frac{x_{2}}{h_{\infty}}, \\
& \bar{p}=\frac{h_{\infty}^{n+1} p}{k U^{n} \ell}, \quad \overline{\boldsymbol{\tau}}=\frac{h_{\infty}^{n+1} \boldsymbol{\tau}}{k U^{n} \ell}, \quad \overline{\dot{\gamma}}=\frac{h_{\infty} \dot{\gamma}}{U}, \quad \bar{h}\left(\bar{x}_{1}\right)=\frac{h\left(x_{1}\right)}{h_{\infty}},
\end{aligned}
$$

where we anticipate the form of the shear-thinning viscosity, $\eta(\dot{\gamma})=$ $k|\dot{\gamma}|^{n-1}$ and the scaling of the shear rate $|\dot{\gamma}|=\left|\partial u_{1} / \partial x_{2}\right| \approx U / h_{\infty}$. The limit $n=1$ corresponds to a Newtonian fluid. Three dimensionless parameters enter the nondimensional lubrication equations and boundary conditions for this flow problem in the limit that inertia is negligible:

$$
\mathcal{C}=\frac{k U^{n} \ell^{3}}{\sigma h_{\infty}^{n+2}}, \quad \mathcal{G}=\frac{\rho g h_{\infty}^{n+1}}{k U^{n}}, \quad \mathcal{E}=\frac{N U^{n} h_{\infty}^{1-n}}{k \ell}
$$

where $\mathcal{C}$ is a capillary number, and $\mathcal{G}$ represents the ratio of gravitational to viscous shear stresses. The third dimensionless number, a Weissenberg number $\mathcal{E}$, enters the problem via the constitutive equation; $N$ is a material constant related to the first normal stress coefficient $\Psi_{1}(\dot{\gamma})=N|\dot{\gamma}|^{2 n-2}$. The Weissenberg number $\mathcal{E}$ represents the ratio of first normal stress differences to the viscous shear stresses, and reflects the importance of elasticity in the flow. We shall refer to a flow in which $\mathcal{E} \ll 1$ as weakly elastic and one in which $\mathcal{E} \gg 1$ as strongly elastic.

Although the parameters $\mathcal{C}, \mathcal{E}$ and $\mathcal{G}$ are useful in representing the nondimensional form of the equations, they are clearly not useful for expressing our final results since they are defined in terms of the unknowns $h_{\infty}$ and $\ell$, both of which depend on the rotation rate or substrate withdrawal speed. Therefore although we shall use these parameters in manipulating the equations, we shall ultimately write our results in terms of the following related nondimensional parameters which do not 
involve the unknown dynamic length-scales $h_{\infty}$ and $\ell$ :

$$
\tilde{\mathcal{C}}=3 \frac{k U^{n}}{\sigma \kappa_{m}^{1-n}}, \quad \tilde{\mathcal{E}}=\frac{N U^{n} \kappa_{m}^{n}}{k} .
$$

The parameters $\tilde{\mathcal{C}}$ and $\tilde{\mathcal{E}}$ are, respectively, modified capillary and modified Weissenberg numbers. The problem thus reduces to determining the variation in the dimensionless scales $h_{\infty} \kappa_{m}$ and $\ell \kappa_{m}$ as a function of $\tilde{\mathcal{C}}, \tilde{\mathcal{E}}$. The final theoretical predictions written in terms of the parameters $\tilde{\mathcal{C}}$ and $\tilde{\mathcal{E}}$ can be compared to the experimental results.

In the lubrication limit, $h_{\infty} / \ell \ll 1$, and when inertia is negligible in the sense that $\rho U^{2-n} h_{\infty}^{n+1} /(k \ell) \ll 1$, the momentum equations (3) are well approximated by the nondimensional lubrication equations for creeping flow:

$$
\begin{aligned}
\frac{\partial \bar{u}_{1}}{\partial \bar{x}_{1}}+\frac{\partial \bar{u}_{2}}{\partial \bar{x}_{2}} & =0 \\
\frac{\partial \bar{\tau}_{11}}{\partial \bar{x}_{1}}+\frac{\ell}{h_{\infty}} \frac{\partial \bar{\tau}_{21}}{\partial \bar{x}_{2}}-\mathcal{G} \sin \alpha & =O\left(\frac{h_{\infty}}{\ell}\right), \\
\frac{\partial \bar{\tau}_{22}}{\partial \bar{x}_{2}}+\frac{h_{\infty}}{\ell} \mathcal{G} \cos \alpha & =O\left(\frac{h_{\infty}}{\ell}\right) .
\end{aligned}
$$

In terms of nondimensional variables, the boundary conditions (4) are

$$
\overline{\mathbf{u}}\left(\bar{x}_{1}, 0\right)=\mathbf{e}_{1}, \quad \mathbf{n} \cdot \overline{\boldsymbol{\tau}}=\left(-\bar{p}_{0}+\mathcal{C}^{-1} \bar{\kappa}\right) \mathbf{n} \quad \text { on } \bar{x}_{2}=\bar{h}\left(\bar{x}_{1}\right),
$$

where $\bar{\kappa}=\ell^{2} \kappa / h_{\infty}$. In order to solve equations (8a-c), we require information from a constitutive equation, and the Criminale-EricksenFilbey constitutive equation is introduced next.

\subsection{Criminale-Ericksen-Filbey Constitutive Equation}

In this section we derive the pressure and velocity fields in the fluid film when the fluid rheology can be represented by the CriminaleEricksen-Filbey constitutive equation (1). Since the magnitude of the second normal stress coefficient $\left|\Psi_{2}(\dot{\gamma})\right|$ is usually much smaller than the magnitude of the first normal stress coefficient $\left|\Psi_{1}(\dot{\gamma})\right|$ in polymer solutions or moderately entangled polymer melts (Bird et al., 1987), we make the approximation $\left|\Psi_{2}(\dot{\gamma}) / \Psi_{1}(\dot{\gamma})\right| \approx 0$. Using the nondimensional variables defined in (5) and also

$\bar{\eta}(\bar{\gamma})=\frac{h_{\infty}^{n-1} \eta(\dot{\gamma})}{k U^{n-1}}, \quad \bar{\Psi}_{1}(\bar{\gamma})=\frac{h_{\infty}^{2 n-2} \Psi_{1}(\dot{\gamma})}{N U^{2 n-2}}, \quad \bar{\Psi}_{2}(\overline{\dot{\gamma}}) \approx 0, \quad \overline{\dot{\gamma}}=\frac{h_{\infty} \dot{\gamma}}{U}$, 
the nondimensional $\mathrm{CEF}$ equation takes the form

$$
\overline{\boldsymbol{\tau}}=-\bar{p} \mathbf{I}+\frac{h_{\infty}}{\ell} \bar{\eta}(\overline{\dot{\gamma}}) \overline{\dot{\gamma}}-\frac{\mathcal{E}}{2} \bar{\Psi}_{1}(\overline{\dot{\gamma}}) \overline{\dot{\gamma}}_{(1)} .
$$

From this point onwards, we use nondimensional variables unless indicated otherwise and therefore we no longer use overbars.

In the lubrication approximation the leading-order contributions to the rate of deformation tensor and each component of its first convected derivative are

$$
\dot{\gamma}=\frac{\partial u_{1}}{\partial x_{2}} \mathbf{e}_{2} \mathbf{e}_{1}, \quad \dot{\gamma}_{(1)}=-2\left(\frac{\partial u_{1}}{\partial x_{2}}\right)^{2} \mathbf{e}_{1} \mathbf{e}_{1}+\frac{h_{\infty}}{\ell} T(\mathbf{u})\left(\mathbf{e}_{1} \mathbf{e}_{2}+\mathbf{e}_{2} \mathbf{e}_{1}\right),(1
$$

where $T(\mathbf{u})=(\mathbf{u} \cdot \nabla) \frac{\partial u_{1}}{\partial x_{2}}+2 \frac{\partial u_{1}}{\partial x_{1}} \frac{\partial u_{1}}{\partial x_{2}}$. Therefore the CEF constitutive equation reduces to

$\boldsymbol{\tau}=\left(\begin{array}{ccc}-p+\mathcal{E} \Psi_{1}(\dot{\gamma})\left(\frac{\partial u_{1}}{\partial x_{2}}\right)^{2} & \frac{h_{\infty}}{\ell} \eta(\dot{\gamma}) \frac{\partial u_{1}}{\partial x_{2}}-\frac{\mathcal{E} h_{\infty}}{2 \ell} \Psi_{1}(\dot{\gamma}) T(\mathbf{u}) & 0 \\ \frac{h_{\infty}}{\ell} \eta(\dot{\gamma}) \frac{\partial u_{1}}{\partial x_{2}}-\frac{\mathcal{E} h_{\infty}}{2 \ell} \Psi_{1}(\dot{\gamma}) T(\mathbf{u}) & -p & 0 \\ 0 & 0 & -p\end{array}\right)$

Substituting (13) into the lubrication equations (8b,c) and neglecting terms $O\left(h_{\infty} / \ell\right)$ yields

$$
\begin{aligned}
& -\frac{\partial p}{\partial x_{2}}+\frac{h_{\infty}}{\ell} \mathcal{G} \cos \alpha=0, \\
& -\frac{\partial p}{\partial x_{1}}+\mathcal{E} \frac{\partial}{\partial x_{1}}\left[\Psi_{1}(\dot{\gamma})\left(\frac{\partial u_{1}}{\partial x_{2}}\right)^{2}\right]+\frac{\partial}{\partial x_{2}}\left[\left(\eta(\dot{\gamma}) \frac{\partial u_{1}}{\partial x_{2}}\right)-\frac{1}{2} \mathcal{E} \Psi_{1}(\dot{\gamma}) T(\mathbf{u})\right] \\
& -\mathcal{G} \sin \alpha=0 .
\end{aligned}
$$

Equations very similar to these formed the basis of analysis of fiber coating by de Ryck and Quéré (1998). Solving (14a) for the pressure subject to the boundary condition (9) gives

$$
p\left(x_{1}, x_{2}\right)=p_{0}-\mathcal{C}^{-1} \kappa+\frac{h_{\infty}}{\ell} \mathcal{G}\left(x_{2}-h\right) \cos \alpha,
$$

and substituting (15) for the pressure into (14b) leads to the equation

$$
\begin{aligned}
\mathcal{C}^{-1} \frac{d \kappa}{d x_{1}}+ & \mathcal{E} \frac{\partial}{\partial x_{1}}\left[\Psi_{1}(\dot{\gamma})\left(\frac{\partial u_{1}}{\partial x_{2}}\right)^{2}\right]+\frac{\partial}{\partial x_{2}}\left[\left(\eta(\dot{\gamma}) \frac{\partial u_{1}}{\partial x_{2}}\right)\right. \\
& \left.-\frac{1}{2} \mathcal{E} \Psi_{1}(\dot{\gamma}) T(\mathbf{u})\right]-\mathcal{G} \sin \alpha+\frac{h_{\infty}}{\ell} \mathcal{G} \frac{d h}{d x_{1}} \cos \alpha=0 .
\end{aligned}
$$


Equation (16) is to be solved for the velocity field $u_{1}\left(x_{1}, x_{2}\right)$ which also satisfies the continuity equation (8a) and the boundary conditions (9). The solution can only be determined after we assume explicit forms for the viscosity $\eta(\dot{\gamma})$ and the first normal stress coefficient $\Psi_{1}(\dot{\gamma})$. Here we take the dimensional functional forms

$$
\eta=k|\dot{\gamma}|^{n-1}, \quad \Psi_{1}=N|\dot{\gamma}|^{2 n-2} .
$$

The constants $k, n$ and $N$ are determined from rheological data to capture the power-law dependence of the viscosity on the shear rate at high shear rates. Note that the constant $N$ is a factor of $3 / 2$ larger than the constant $N$ in the theory of de Ryck and Quéré (1998).

The forms of the exponents for the shear-rate dependence of viscosity and first normal stress coefficient, both involving $n$, have been chosen in accordance with the common experimental observation that $\Psi_{1}(\dot{\gamma}) \propto \eta(\dot{\gamma})^{2}$. This choice can be motivated by considering the convected derivative term $(\nabla \mathbf{u})^{T} \cdot \boldsymbol{\tau}$ in the constitutive equation, which results in a term of the form $\lambda \dot{\gamma} \tau_{12}$ entering the equation for $\tau_{11}$, where $\lambda$ is the relaxation time of the polymer. As a consequence, $\tau_{11} \approx \lambda \dot{\gamma}^{2} \eta(\dot{\gamma})$ is second-order in shear rate since $\tau_{12} \approx \eta(\dot{\gamma}) \dot{\gamma}$. Finally kinetic theory for polymer solutions shows that $\eta(\dot{\gamma}) \approx \lambda G$ where $G$ is the shear modulus, and therefore we expect $\tau_{11}-\tau_{22} \approx \eta(\dot{\gamma})^{2} \dot{\gamma}^{2} / G$. This result is also consistent with experimental observations (Barnes et al., 1989)), and with the functional form of the White-Metzner constitutive equation (Bird et al., 1987).

\subsection{Derivation of the THIN-FILM EQUATION}

In this section we use the continuity equation (8a) and equation (16) to derive the thin-film equation which characterizes the film thickness $h\left(x_{1}\right)$. We generalize the solution method of de Ryck and Quéré (1998), who analyzed analogous equations for a viscoelastic constitutive equation in the fiber geometry. In order to find an approximate solution to equation (16) and to achieve this generality, we approximate the velocity field using the general form

$$
u_{1}\left(x_{1}, x_{2}\right)=1+A\left(x_{1}\right)\left(\frac{x_{2}^{2}}{2}-x_{2} h\right),
$$

which satisfies the boundary conditions (9) and is motivated by the quadratic dependence of the velocity field on $x_{2}$ in a Newtonian flow. When the viscosity is shear thinning, the velocity field is no longer quadratic; however, a more accurate form is not analytically tractable in an analysis that is general to both the weakly and strongly elastic limits of the CEF equation. 
By integrating the continuity equation (8a) over the film depth, we find that the flux $q$ defined by

$$
q=\int_{0}^{h\left(x_{1}\right)} u_{1} \mathrm{~d} x_{2}
$$

must be constant, as is consistent with the steady-state profile. This flux is nondimensional, scaled by $U h_{\infty}$. As $x_{1} \rightarrow \infty, u \rightarrow 1, h \rightarrow 1$ and therefore the nondimensional flux $q \rightarrow 1$ as $x \rightarrow \infty$. Since the flux is constant, $q=1$ everywhere.

In order that the flux obtained from the assumed form of the velocity field (18) is constant and equal to unity, $A\left(x_{1}\right)$ must take the form

$$
A\left(x_{1}\right)=\frac{3\left[h\left(x_{1}\right)-1\right]}{h\left(x_{1}\right)^{3}} .
$$

Using (20) and substituting the assumed form of the velocity field (18) into equation (16) yields the following thin-film equation:

$$
\begin{gathered}
\mathcal{C}^{-1} \frac{d \kappa}{d x_{1}}+\mathcal{E}\left\{\frac{\partial}{\partial x_{1}}\left[A\left(h-x_{2}\right)\right]^{2 n}-\frac{1}{2} \frac{\partial}{\partial x_{2}}\left[\left[A\left(h-x_{2}\right)\right]^{2 n-2} T\left(\mathbf{u}\left(A, h, x_{2}\right)\right)\right]\right\} \\
-\frac{\partial}{\partial x_{2}}\left[A\left(h-x_{2}\right)\right]^{n}-\mathcal{G} \sin \alpha+\frac{h_{\infty}}{\ell} \mathcal{G} \frac{d h}{d x_{1}} \cos \alpha=0 .
\end{gathered}
$$

Note that we retain the $O\left(h_{\infty} \mathcal{G} / \ell\right)$ term which is important in the static meniscus.

We now average the elastic stress and the viscous stress gradient terms across the film depth, i.e. in the $x_{2}$-direction. Specifically, in the case of the elastic stress term, we approximate $\left[A\left(h-x_{2}\right)\right]^{n}$ by $h^{-1} \int_{0}^{h}\left[A\left(h-x_{2}\right)\right]^{n} \mathrm{~d} x_{2}$. The average of the term involving $T\left(\mathbf{u}\left(A, h, x_{2}\right)\right)$ in (21) equals $-\frac{1}{2} \mathcal{E} A^{2 n-1} h^{2 n-2}$. After averaging, the equation governing $h\left(x_{1}\right)$ is

$$
\begin{array}{r}
\mathcal{C}^{-1} \frac{d \kappa}{d x_{1}}+\mathcal{E} \frac{3^{2 n-1}[(10 n-1) h-12 n](2-h)(h-1)^{2 n-2}}{2(2 n+1) h^{4 n+1}} \frac{d h}{d x_{1}} \\
+\frac{3^{n}(h-1)^{n}}{h^{2 n+1}}-\mathcal{G} \sin \alpha+\frac{h_{\infty}}{\ell} \mathcal{G} \frac{d h}{d x_{1}} \cos \alpha=0,
\end{array}
$$

which must be solved subject to the boundary condition $h\left(x_{1}\right) \rightarrow 1$ as $x_{1} \rightarrow \infty$.

\subsection{Asymptotic Results for the Weakly elastic limit: $\mathcal{E} \ll 1$}

In the limit $\mathcal{E} \ll 1$, the elastic stress is negligible in comparison to the viscous shear stress and we refer to this as the weakly elastic limit. 
In terms of the dimensionless parameters $\tilde{\mathcal{C}}$ and $\tilde{\mathcal{E}}$ that do not involve the actual film thickness but only utilize external scales and material properties, we show below that the condition $\mathcal{E} \ll 1$ may be written as $\tilde{\mathcal{E}}^{2 n+1} \ll \tilde{\mathcal{C}}^{2 n-1}$. For the power-law form of the viscosity $\eta(\dot{\gamma})=k|\dot{\gamma}|^{n-1}$ considered in this work, the weakly elastic limit encompasses both the Newtonian limit, with $n=1$, and the power-law viscosity limit, with $0<n<1$.

The thickness of the thin film may be determined from equation (22) using matched asymptotics. Firstly, we note that the definition of $\mathcal{C}$ included the parameter $\ell$ which is not yet specified. We now choose $\ell=\left(\sigma h_{\infty}^{n+2} /\left(3 k U^{n}\right)\right)^{1 / 3}$ so that $\mathcal{C}=1 / 3^{n}$. Equation (22) is then approximated by

$$
\frac{d^{3} h}{d x_{1}^{3}}+\frac{(h-1)^{n}}{h^{2 n+1}}+O\left(\frac{h_{\infty}}{\ell}, \mathcal{E}, \mathcal{G}\right)=0 .
$$

Here the curvature $\kappa$ has been approximated by $h^{\prime \prime}+O\left(h_{\infty} / \ell\right)$. Although as remarked in section 2.1 the exact expression for the curvature varies according to the geometry, this leading-order approximation is valid for all geometries.

Equation (23) describes the film thickness $h\left(x_{1}\right)$ in the dynamic meniscus. Note that the equation contains no parameters other than $n$, and it is subject to the boundary condition $h\left(x_{1}\right) \rightarrow 1$ as $x_{1} \rightarrow \infty$. For a specified value of $n$, the equation can be integrated backwards numerically from a large value of $x_{1}$, from the flat film towards the meniscus, starting from a small perturbation of the uniform film. As $x_{1}$ decreases, a constant value of the curvature $\kappa \approx h^{\prime \prime}$ is eventually attained, which is a function of $n$ only:

$$
\frac{d^{2} h}{d x_{1}^{2}}\left(x_{1} \rightarrow-\infty\right)=f(n)
$$

This value of the curvature must match to the curvature of the static meniscus.

We now rescale variables to capture the dominant balance in the static meniscus. Introducing nondimensional variables $H$ and $X_{1}$ defined by:

$$
h=\frac{H}{h_{\infty} \kappa_{m}}, \quad x_{1}=\frac{X_{1}}{\ell \kappa_{m}},
$$

the thin-film equation (22) becomes

$$
\frac{d \kappa}{d X_{1}}-\sin \alpha+\frac{d H}{d X_{1}} \cos \alpha+O\left(\frac{\mathcal{E} h_{\infty}^{2 n} \ell \kappa_{m}^{2 n+1}}{\mathcal{G}}, \frac{\left(h_{\infty} \kappa_{m}\right)^{n+1}}{\mathcal{G}}\right)=0
$$


This equation characterizes the static meniscus (for details, see Wilson 1981). It is necessary to retain the full nonlinear curvature to describe the entire meniscus region accurately, but in the matching region the curvature is approximated by the second derivative of $H\left(X_{1}\right)$ and is equal to the curvature of the dynamic meniscus:

$$
1=\frac{d^{2} H}{d X_{1}^{2}}=\frac{h_{\infty}}{\ell^{2} \kappa_{m}} \frac{d^{2} h}{d x_{1}^{2}}=\frac{h_{\infty}}{\ell^{2} \kappa_{m}} f(n) \quad \text { in the matching region. }
$$

Eliminating $\ell$ from (27) shows that the film thickness is predicted to be

$$
\frac{h_{\infty}}{\kappa_{m}^{-1}}=\left[9 f(n)^{3}\left(\frac{k U^{n}}{\sigma \kappa_{m}^{1-n}}\right)^{2}\right]^{1 /(2 n+1)}=\left[9 f(n)^{3} \tilde{\mathcal{C}}^{2}\right]^{1 /(2 n+1)} .
$$

This result is the first term in a series; the next term is expected to be of order $\tilde{\mathcal{C}}^{3 /(2 n+1)}$. Although the CEF equation in the weakly elastic limit is equivalent to the power-law constitutive equation, use of the approximate velocity field (18) to solve the thin-film equation (22) and determine the film thickness leads to a small deviation from the exact result of the power-law constitutive equation, which is discussed in Appendix A.

The function $f(n)$ has been calculated numerically for a range of values of $n$ and is plotted in Figure 2. When $n=1$, equation (28) reduces to $h_{\infty}=1.34(k U / \sigma)^{2 / 3} \kappa_{m}^{-1}$ in agreement with classical results (Bretherton, 1961; Derjaguin, 1943; Landau and Levich, 1942). Gutfinger and Tallmadge (1965) used a numerical fitting procedure and found that the function $f(n)$ was well approximated by $0.646-0.76 \ln n$, which is indicated in the inset to Figure 2. Note that for completeness we extend this computation for $n>1$ corresponding to shear-thickening fluids. Such fluids are typically hard to formulate experimentally, although some concentrated suspensions do exhibit shear thickening (Barnes et al., 1989).

We consider the restrictions on the validity of the result (28). Substituting the value of $h_{\infty}$ into the expressions for $\ell$ and $\mathcal{G}$ yields

$$
\ell \approx \tilde{\mathcal{C}}^{1 /(2 n+1)} \kappa_{m}^{-1}, \quad \frac{h_{\infty}}{\ell} \approx \tilde{\mathcal{C}}^{1 /(2 n+1)}, \quad \text { and } \quad \mathcal{G} \approx \frac{\rho g}{\sigma \kappa_{m}^{2}} \tilde{\mathcal{C}}^{1 /(2 n+1)},
$$

where $\approx$ indicates that the effect of the numerical prefactor involving $f(n)$ has not been accounted for. Therefore the condition $\tilde{\mathcal{C}} \ll 1$ is equivalent to both the requirements that $h_{\infty} / \ell \ll 1$ and that the $O(\mathcal{G})$ gravity term in $(23)$ is negligible. (Note that $\rho g /\left(\sigma \kappa_{m}^{2}\right)<1$ provided a plate or roller is withdrawn at an angle $\alpha<2 \pi / 3$ and this inequality also holds for a fiber withdrawn vertically.) We have 


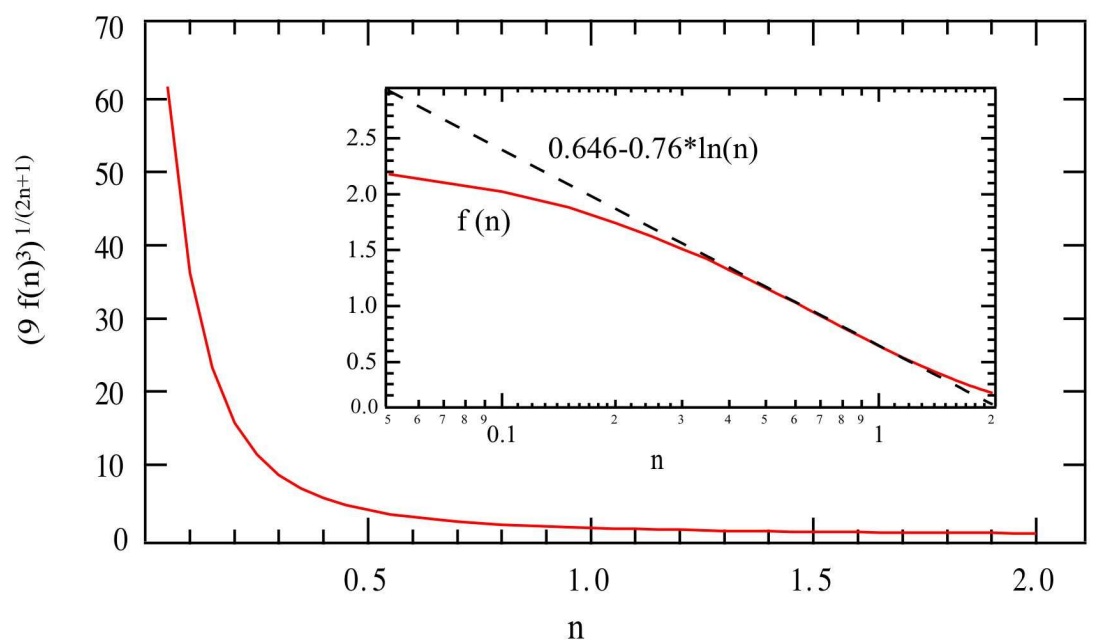

Figure 2. Plot of the prefactor $\left(9 f(n)^{3}\right)^{1 /(2 n+1)}$ for the predicted film thickness in the weakly elastic limit, as defined in equation (24). The inset shows $f(n)$ versus $n$ (solid line) and the approximation $0.646-0.76 \ln n$ (Gutfinger and Tallmadge, 1965).

also assumed that $\mathcal{E} \ll 1$. Using the prediction for the film thickness (28) in the definition of $\mathcal{E}$ and rewriting this condition in terms of the parameters $\tilde{\mathcal{E}}$ and $\tilde{\mathcal{C}}$ which do not depend on the film thickness, we find that $\mathcal{E} \approx \tilde{\mathcal{E}} / \tilde{\mathcal{C}}^{(2 n-1) /(2 n+1)}$ and therefore the condition $\mathcal{E} \ll 1$ can be rewritten as $\tilde{\mathcal{E}}^{2 n+1} \ll \tilde{\mathcal{C}}^{2 n-1}$.

\subsection{ASYMPTOTIC RESUlTS FOR THE STRONGLY ELASTIC LIMIT: $\mathcal{E} \gg 1$}

When $\mathcal{E} \gg 1$, the elastic stresses dominate over the viscous shear stresses and we refer to this as the strongly elastic limit. We shall show that the condition $\mathcal{E} \gg 1$ is equivalent to $\tilde{\mathcal{E}}^{2 n+1} \gg \tilde{\mathcal{C}}^{2 n-1}$. To predict the film thickness in this limit, we use matched asymptotic analysis as in section 2.4 but we choose a different scaling for $\ell=$ $\left(\sigma h_{\infty}^{2 n+1} /\left(3 N U^{2 n}\right)\right)^{1 / 2}$. Then $\mathcal{C} \mathcal{E}=1 / 3$ and the thin-film equation $(22)$ reduces to

$$
\begin{array}{r}
\frac{d^{3} h}{d x_{1}^{3}}+\frac{3^{2 n-2}[(10 n-1) h-12 n](2-h)(h-1)^{2 n-2}}{2(2 n+1) h^{4 n+1}} \frac{d h}{d x_{1}} \\
+O\left(\frac{h_{\infty}}{\ell}, \frac{1}{\mathcal{E}}, \frac{\mathcal{G}}{\mathcal{E}}\right)=0,
\end{array}
$$

where again the curvature $\kappa$ is approximated by $h^{\prime \prime}+O\left(h_{\infty} / \ell\right)$. For a given value of $n$ this equation can be integrated backwards numerically 


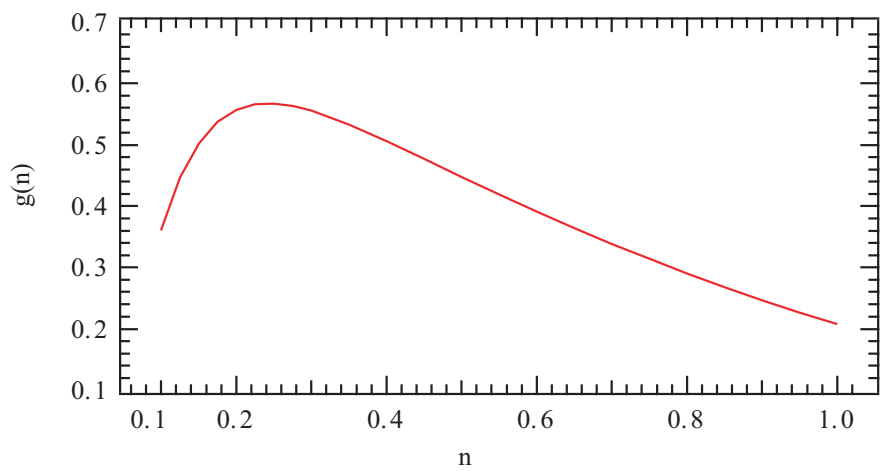

Figure 3. Plot of the prefactor $g(n)$ for the predicted film thickness in the strongly elastic limit, as defined in equation (31).

until a constant value of the curvature, which we denote by the function $g(n)$, is attained. The coefficient multiplying $\frac{d h}{d x_{1}}$ diverges as $h \rightarrow 1$ and therefore must be truncated; physically, this reflects the finite value of the first normal stress coefficient at low shear rates. The results are not sensitive to the details of the truncation.

We now discuss the matching between the dynamic and static menisci. To analyze the static meniscus we use the same rescaling as in the weakly elastic case, given by (25), although now the definitions of $h_{\infty}$ and $\ell$ are different. This rescaling again leads to equation (26), and

$$
1=\frac{d^{2} H}{d X_{1}^{2}}=\frac{h_{\infty}}{\kappa_{m} \ell^{2}} \frac{d^{2} h}{d x_{1}^{2}}=\frac{h_{\infty}}{\ell^{2} \kappa_{m}} g(n) \quad \text { in the matching region. }
$$

Eliminating $\ell$ from equation (31) leads to the following prediction for the film thickness:

$$
h_{\infty}=\left(3 g(n) \frac{N}{\sigma \kappa_{m}}\right)^{1 / 2 n} U=(g(n) \tilde{\mathcal{C}} \tilde{\mathcal{E}})^{1 / 2 n} \kappa_{m}^{-1} .
$$

In this case, the full result is a series in the parameter $(\tilde{\mathcal{C}} \tilde{\mathcal{E}})^{1 / 4 n}$ and the result (32) is the leading-order term. A plot of the function $g(n)$ for a range of values of $n$ appears in Figure 3.

From the conditions $\tilde{\mathcal{C}} \tilde{\mathcal{E}} \ll 1$ and $\tilde{\mathcal{C}}^{2 n-1} \ll \tilde{\mathcal{E}}^{2 n+1}$ we find that $(\tilde{\mathcal{C}} \tilde{\mathcal{E}})^{1 / 2 n} \gg \tilde{\mathcal{C}}^{2 n /(2 n+1)}$ and therefore the film thickness in the strongly elastic limit of the CEF equation is predicted to be larger than in the weakly elastic limit. Physically, the film thickness is increased relative to the film thickness of a weakly elastic fluid because the first normal stress difference generates a net stress perpendicular to the substrate surface and also leads to an effective tension in the streamlines, which both tend to increase the film thickness. 
Table II. Summary of theoretical predictions for film thickness $h_{\infty}$ and the conditions for validity in the weakly elastic and strongly elastic limits, written in terms of the external dimensional variables in the problem. In both limits, substitution of the expressions for $h_{\infty}$ and $\ell$ into equation (6) convert the restriction on $\mathcal{E}$ into a condition on $\tilde{\mathcal{E}}^{2 n+1} / \tilde{\mathcal{C}}^{2 n-1}=N^{2 n+1} U^{2 n} \kappa_{m}^{4 n-1} \sigma^{2 n-1} / k^{4 n}$. The condition that $h_{\infty} / \ell \ll 1$ must be satisfied in order that the lubrication approximation used in the analysis is valid; the requirement that $\rho U^{2-n} h_{\infty}^{n+1} /(k \ell) \ll 1$ results from inertial terms being neglected in the analysis. The expressions for $\ell$ in the weakly and strongly elastic limits are given in equations (29) and (33) respectively.

\begin{tabular}{lll}
\hline & $\begin{array}{l}\text { predicted scaling } \\
\text { of film thickness } h_{\infty}\end{array}$ & conditions for validity \\
\hline $\begin{array}{l}\text { weakly } \\
\text { elastic } \\
\mathcal{E} \ll 1\end{array}$ & $h_{\infty} \approx\left(\frac{k U^{n}}{\sigma}\right)^{2 /(2 n+1)} \kappa_{m}^{-3 /(2 n+1)}$ & $\frac{h_{\infty}}{\ell} \ll 1 \Rightarrow \frac{k U^{n}}{\sigma \kappa_{m}^{1-n}} \ll 1$ \\
& $\frac{\rho U^{2-n} h_{\infty}^{n+1}}{k \ell} \ll 1 \Rightarrow \frac{\rho U^{2} \kappa_{m}^{-1}}{\sigma} \ll 1$ \\
\hline $\begin{array}{l}\text { strongly } \\
\text { elastic }\end{array}$ & $h_{\infty} \approx\left(\frac{N U^{2 n}}{\sigma}\right)^{1 /(2 n)} \kappa_{m}^{-1 /(2 n)}$ & $\frac{h_{\infty} \ll 1 \Rightarrow \frac{N U^{2 n}}{\ell} \ll 1}{\sigma \kappa_{m}^{1-2 n} \ll 1}$ \\
\hline
\end{tabular}

We now discuss the restrictions under which these results are valid. Using equation (32), we find that

$$
\ell \approx(\tilde{\mathcal{C}} \tilde{\mathcal{E}})^{1 / 4 n} \kappa_{m}^{-1}, \quad \frac{h_{\infty}}{\ell} \approx(\tilde{\mathcal{C}} \tilde{\mathcal{E}})^{1 / 4 n}, \quad \text { and } \quad \frac{\mathcal{G}}{\mathcal{E}} \approx \frac{\rho g}{\sigma \kappa_{m}^{2}}(\tilde{\mathcal{C}} \tilde{\mathcal{E}})^{1 / 4 n}
$$

Since we neglected terms of order $h_{\infty} / \ell$ and $\mathcal{G} / \mathcal{E}$ in equation $(30)$, we require that $\left\{h_{\infty} / \ell, \mathcal{G} / \mathcal{E}\right\} \ll 1$. Both of these conditions are satisfied provided $\tilde{\mathcal{C}} \tilde{\mathcal{E}} \ll 1$. Finally since $\mathcal{E} \approx\left[\tilde{\mathcal{E}}^{2 n+1} / \tilde{\mathcal{C}}^{2 n-1}\right]^{1 / 4 n}$ and $\mathcal{E} \gg 1$, we require that $\tilde{\mathcal{C}}^{2 n-1} \ll \tilde{\mathcal{E}}^{2 n+1}$. For completeness we summarize the key results at the end of this section. The film thickness and the conditions of validity, expressed in terms of the external parameters in the problem, for both the weakly and strongly elastic limits are listed in Table II. Figure 4 shows a conceptual diagram indicating the range of validity of the predictions of film thickness in the weakly and strongly elastic limits. The diagrams differ in the two cases $0<n<1 / 2$ and $1 / 2<n<1$ because the exponent of $\mathcal{C}$ in the relationship $\mathcal{E}^{2 n+1}=\mathcal{C}^{2 n-1}$, which quantifies the crossover between weakly and strongly elastic behavior, changes sign when $n=1 / 2$.

We note that one aspect of the theory in the strongly elastic limit is not entirely rigorous: since the CEF model is based on a retarded motion expansion for slowly varying flows its validity is strictly speaking limited to the small Deborah number limit, where the effective Deborah 
(a)

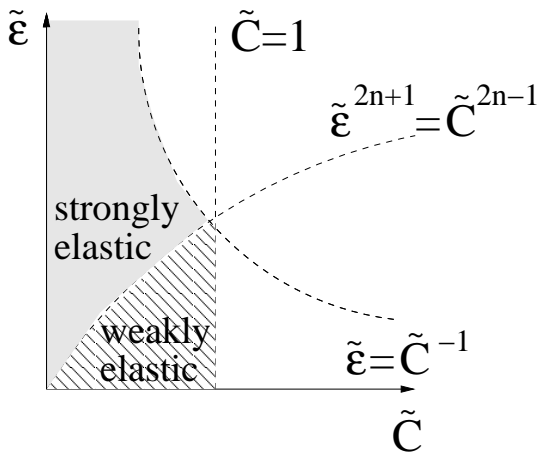

(b)

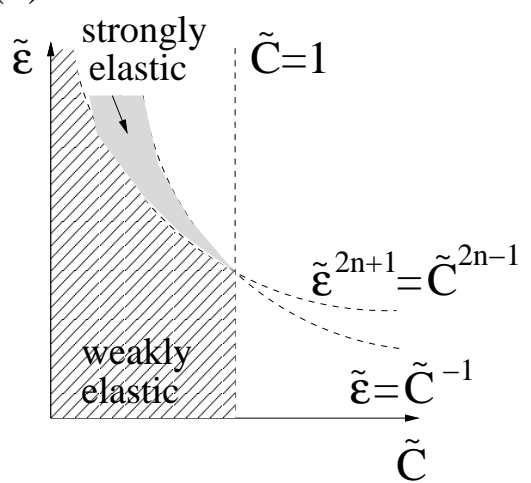

Figure 4. Diagram indicating the range of validity of results in the weakly and strongly elastic limits, (a) for $1 / 2<n<1$ and (b) for $0<n<1 / 2$. The restrictions indicated are those imposed by lubrication theory and by the value of $\mathcal{E}$. The condition imposed by inertia being negligible would have to be represented in a third dimension and is not indicated here.

number $\mathcal{D}_{\text {eff }}$ is the ratio of the polymer relaxation time to the time that a given fluid element is exposed to high shear in the meniscus region, $\mathcal{D}_{\text {eff }}=\lambda U / \ell \approx \lambda U \kappa_{m} /(\tilde{\mathcal{C}} \tilde{\mathcal{E}})^{1 / 4 n}$ (Huilgol, 1975). When the Deborah number is large, extensional effects - which are not accounted for in this theory - may become important. Other constitutive models can be used to capture extensional effects: the CEF model (1) and the specific functional forms (17) of the material functions chosen in the present work are consistent with an asymptotic expansion of the White-Metzner model (Bird et al., 1987) in the limit $\mathcal{D}_{\text {eff }} \ll 1$, but the White-Metzner model retains the full convected nonlinearities which give rise to extensional stresses in the fluid. However, the resulting thin film equations derived from the White-Metzner model are not explicit in the velocity gradient and the complete set of coupled partial differential equations for the velocity field and the shearing and elongational stress components must be solved numerically. We note that a theory similar to that used here was presented by de Ryck and Quéré (1998) for the fiber coating geometry in the strongly elastic limit. The resulting predictions were in good agreement with the corresponding experimental results, and it is therefore possible that extensional effects do not alter the film thickness significantly over a range of $\mathcal{D}_{\text {eff }}$ in other related coating-flow geometries such as those considered here. 


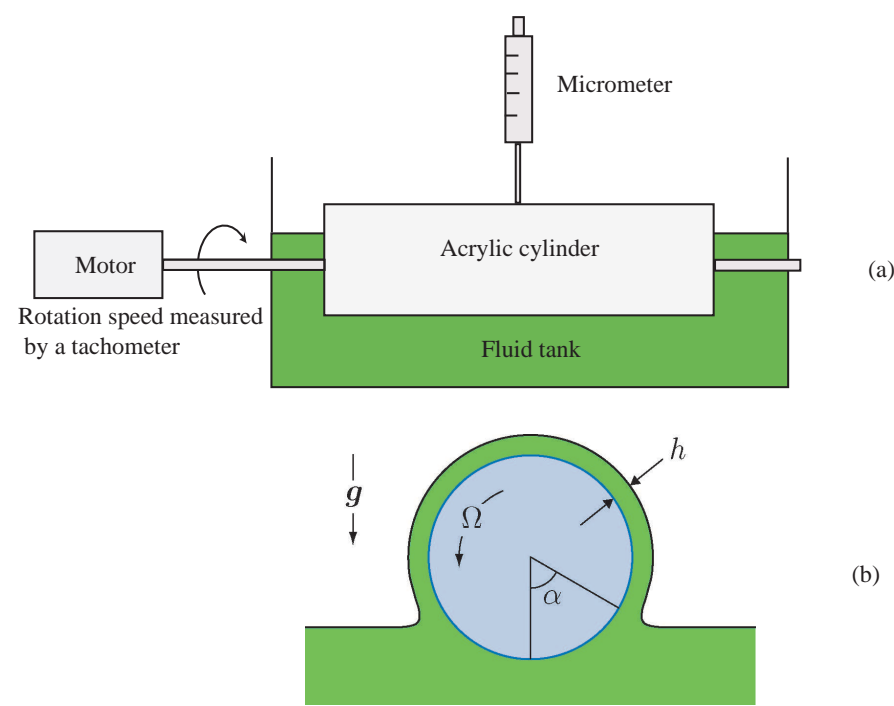

Figure 5. (a) Schematic diagram of the roller coating setup. (b) Sketch of the roller geometry, showing the definition of the inclination angle $\alpha$.

\section{Experimental setup}

In this section we describe the experimental set-up, the rheological measurements for the two fluids and the method used to determine the coating film thickness. A schematic diagram of the system used in the experiments is shown in Figure 5. An acrylic roller with diameter $8.3 \mathrm{~cm}$ and length $26 \mathrm{~cm}$ was partially submerged with its axis oriented horizontally inside a fluid-filled acrylic tank with dimensions of $38 \times 26 \times 13 \mathrm{~cm}^{3}$. The roller was supported at either end by an independently adjustable shaft, which was connected by a belt to interchangeable wheels on a motor. The DC motor could reach a maximum rotation rate of $1400 \mathrm{RPM}$ and had continuous manual dial control. The rotation speed is determined directly from a calibrated digital tachometor installed within the motor unit. Repeated calibrations showed that the measurements based on the tachometer reading agreed with the actual shaft speed to within $0.5 \%$.

The two test fluids used in the experiments were both polymer solutions composed of polyacrylamide (PAA) (from Aldrich) of mass average molecular weight $2.2 \times 10^{6} \mathrm{~g} / \mathrm{mol}$ dissolved in a solvent, consisting of a 50/50 mixture by weight of water and glycerol. The surface tensions of both solutions were measured using a Wilhelmy plate in a Krüss K-10 tensiometer; the value recorded was the static surface tension that is attained when the measurement reached steady state. The solvent viscosity was measured to be $\eta_{s}=6.1 \times 10^{-3}$ Pa.s. One 
fluid contained $0.06 \mathrm{wt} \%$ PAA by weight and the other fluid $1.00 \mathrm{wt} \%$ PAA. The coil overlap concentration $c^{*}$ for the PAA solution can be estimated from the known mass-averaged molecular weight of the chain and the characteristic ratio for the chain (Bird et al., 1987). Using these values, we find $c^{*} \approx 0.5 \mathrm{wt} \%$, depending on the degree of hydrolysis of the PAA, which is expected to be $35 \pm 5 \%$ (Kulicke and Clasen, 2004). Hence the $0.06 \mathrm{wt} \%$ PAA solution is in the dilute regime $\left(c / c^{*}<1\right)$, whereas the $1.00 \mathrm{wt} \%$ solution is in the semi-dilute regime $\left(c / c^{*}>1\right)$. The consequences of these differing concentrations can be observed in the viscometric data reported below. We used a TA Instruments AR1000N stress-controlled rheometer to measure the rheology of the two fluids under both oscillatory and steady shear conditions. All rheological measurements were made at $25^{\circ} \mathrm{C}$. The rheological data for the two fluids is plotted in Figures 6 and 7, and the important rheological parameters and fitted forms of the viscometric functions are summarized in Table III. We discuss the rheological data for each fluid below.

The linear viscoelastic storage and loss moduli, denoted by $G^{\prime}(\omega)$ and $G^{\prime \prime}(\omega)$ respectively, of the $0.06 \mathrm{wt} \%$ PAA solution were measured under small amplitude oscillatory conditions, and the data are plotted for a range of angular frequencies $\omega$ in Figure 6a. Over most of the frequency range measured, $G^{\prime}(\omega)<G^{\prime \prime}(\omega)$ and therefore viscoelastic effects are weak in this fluid. The range of measured data is limited to frequencies $\omega>1 \mathrm{rad} / \mathrm{s}$ because the storage modulus becomes too small to measure accurately at lower frequencies. One estimate of a characteristic relaxation time of the fluid can be obtained using the formula $\lambda=1 / \omega_{\text {crit }}$, where $\omega_{\text {crit }}$ is the value of the frequency at which $G^{\prime}\left(\omega_{\text {crit }}\right)=G^{\prime \prime}\left(\omega_{\text {crit }}\right)$. Using this definition, we find that $\lambda=0.015$ s for this fluid.

The shear viscosity of the $0.06 \mathrm{wt} \%$ fluid was also measured for a range of shear rates under steady shear conditions, and the data is plotted in Figure 6b. We can use a function of the form of the de Ostwaelde power law, $\eta=k|\dot{\gamma}|^{n-1}$, to fit the variation in the viscosity data at large shear rates; the data in the range $|\dot{\gamma}|>7 s^{-1}$ is well described with $n=0.71$ and $k=0.1 \mathrm{~Pa} \cdot \mathrm{s}^{0.71}$. The first normal stress difference of this $0.06 \mathrm{wt} \% \mathrm{PAA} /$ glycerol/water fluid was so small that reliable measurements could not be made over this range of shear rates. However, an approximate estimate of the first normal stress coefficient $\Psi_{10}$ in the limit of zero shear rate can be obtained using the expression $\Psi_{10} \sim 2 \lambda\left(\eta_{0}-\eta_{s}\right)=1.9 \times 10^{-3}$ Pa.s $^{2}$, using the measured value $\eta_{0}=$ 0.067 Pa.s for the zero shear rate viscosity.

The analogous rheological data for the $1.00 \mathrm{wt} \%$ PAA solution is plotted in Figure 7. The storage and loss moduli data shown in Fig- 

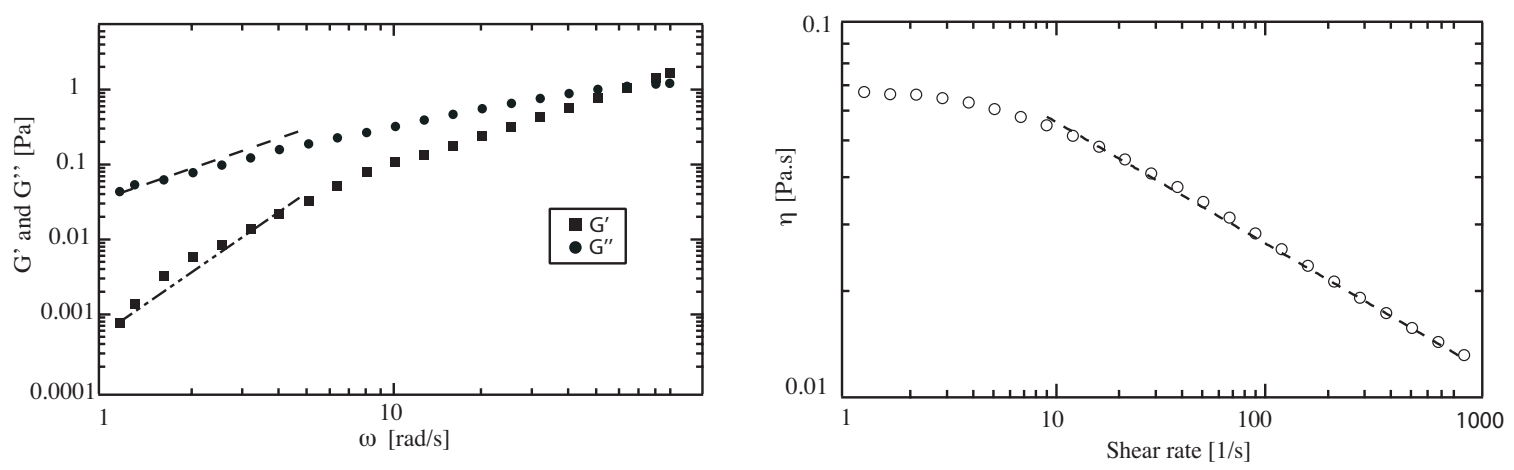

Figure 6. Rheological data for the test fluid containing $0.06 \mathrm{wt} \%$ PAA. (a) Storage $\left(G^{\prime}\right)$ and loss $\left(G^{\prime \prime}\right)$ moduli data as a function of frequency measured under small amplitude oscillatory conditions. The two lines indicated at low frequencies show the asymptotic variations $G^{\prime} \sim \omega, G^{\prime \prime} \sim \omega^{2}$ expected for simple Maxwell fluids in the limit of low frequency. (b) Viscosity data as a function of shear rate measured under steady shear conditions. The zero shear-rate viscosity $\eta_{0}=0.067 \mathrm{~Pa}$.s. The viscosity data at large shear rates, $|\dot{\gamma}|>10 \mathrm{~s}^{-1}$, is fitted using the power-law model $\eta=0.1|\dot{\gamma}|^{-0.29}$ (broken line), where $\dot{\gamma}$ has units $\mathrm{s}^{-1}$.

ure $7 \mathrm{a}$ are such that, over the most of the frequency range measured, $G^{\prime}(\omega)>G^{\prime \prime}(\omega)$ and therefore viscoelastic effects are strong in this fluid. The relaxation time $\lambda=1 / \omega_{\text {crit }}$ is calculated to be $\lambda=3.3 \mathrm{~s}$. The data for the viscosity and first normal stress difference as a function of shear rate measured under steady shear conditions are plotted in Figure 7b. Again, a function of the form $\eta=k|\dot{\gamma}|^{n-1}$ is used to fit the powerlaw behavior of the viscosity data, and a good fit is achieved at all measured shear rates with $n=0.45$ and $k=60 \mathrm{~Pa} . \mathrm{s}^{0.45}$. Over the range of shear rates for which the first normal stress difference is measured, the data can be fitted using a power-law function $\left(\tau_{11}-\tau_{22}\right)=N|\dot{\gamma}|^{2 n}$ where $N=40$ Pa.s ${ }^{0.90}$. The first normal stress difference in the limit of zero shear rate is $\Psi_{10}=\lim _{\dot{\gamma} \rightarrow 0}\left(\tau_{11}-\tau_{22}\right) / \dot{\gamma}^{2} \sim 770$ Pa.s ${ }^{2}$, while the estimate $2 \lambda \eta_{0}=1400 \mathrm{~Pa} . \mathrm{s}^{2}$ is obtained based on the measured value of the zero shear viscosity $\eta_{0}=210$ Pa.s.

We next describe the measurements made in the single roll coating experiments. When the cylinder is partially immersed in the fluid and is rotated about its axis, various different types of steady-state behavior are possible. At very low rotation rates, a continuous fluid film is not generated and the rotating cylinder remains dry above a certain height due to dewetting effects; at intermediate rates, a thin axially uniform fluid film is generated which coats the entire cylinder and finally, at 
Table III. Summary of the rheological properties of the $0.06 \mathrm{wt} \%$ and $1.00 \mathrm{wt} \%$ PAA solutions used in the single roll coating experiments.

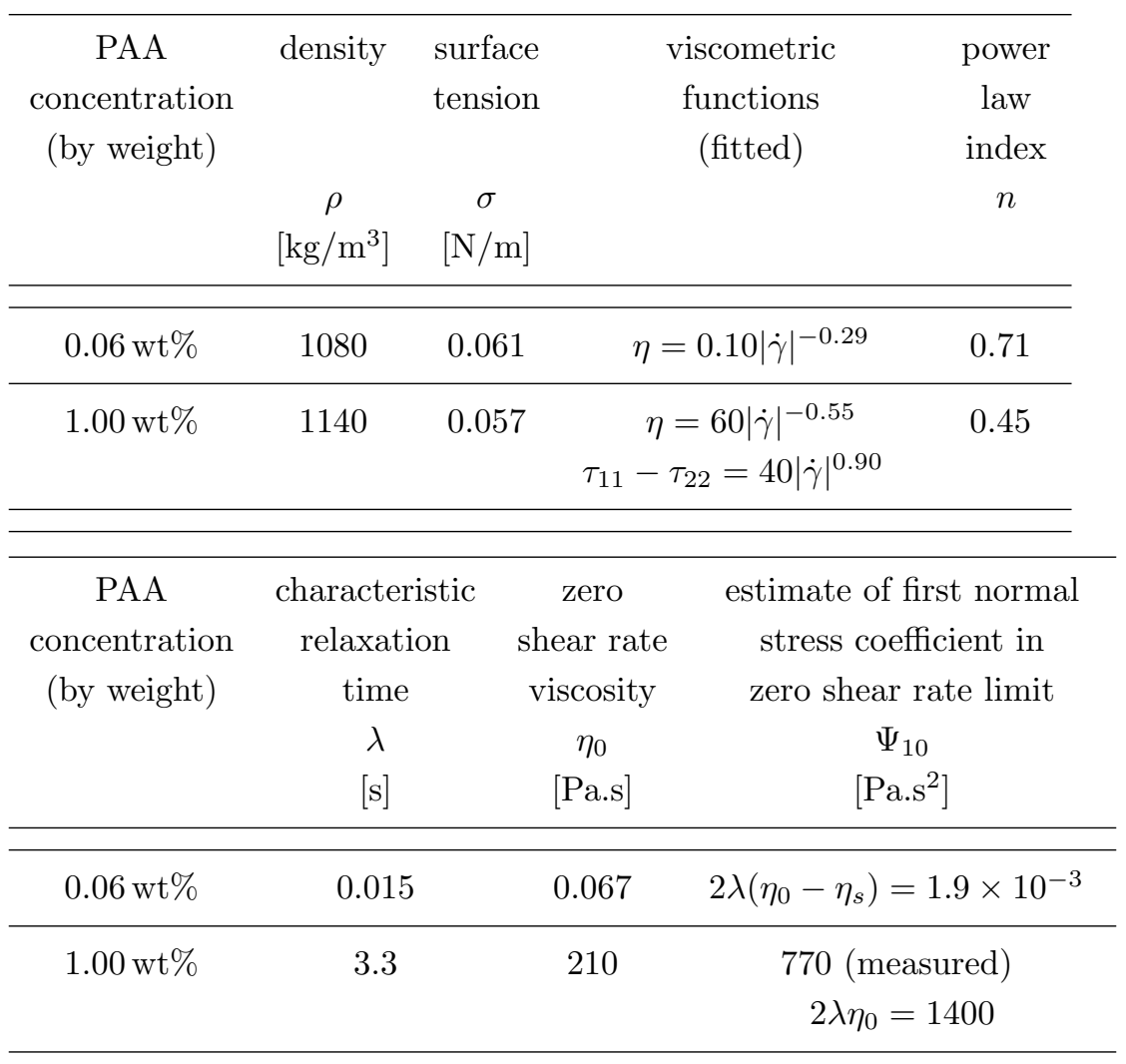

sufficiently high rotation rates an instability develops and the film becomes non-uniform in the axial direction. Our experiments focus on the intermediate range of rotation speeds in which the film thickness is independent of the axial position. The measured film thickness is found to be independent of the rotation direction and, since the cylinder radius is large compared to the capillary length, variations in the film thickness in the azimuthal direction are also negligible. The gravitational terms that cause azimuthal variations in the film thickness are shown to be negligible at leading order away from the meniscus by the asymptotic theory presented in section 2 .

We varied the depth of the fluid in the acrylic tank which corresponds to varying the cylinder immersion depth, defined to be the vertical distance from the flat free surface (away from the curved menis- 
(a)

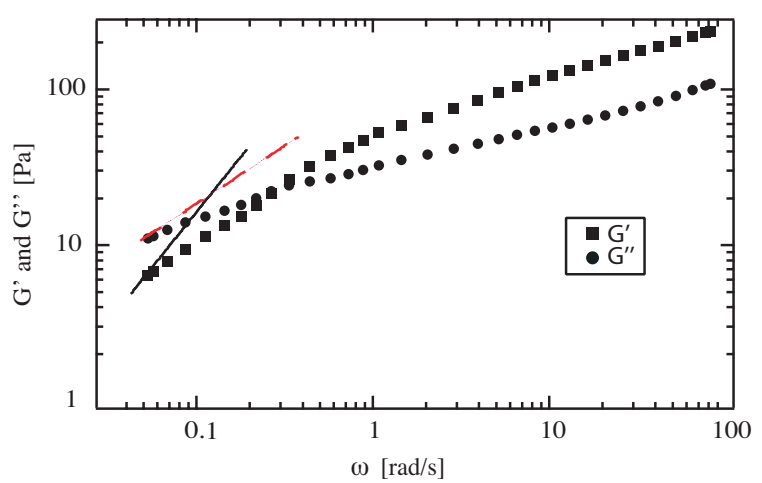

(b)

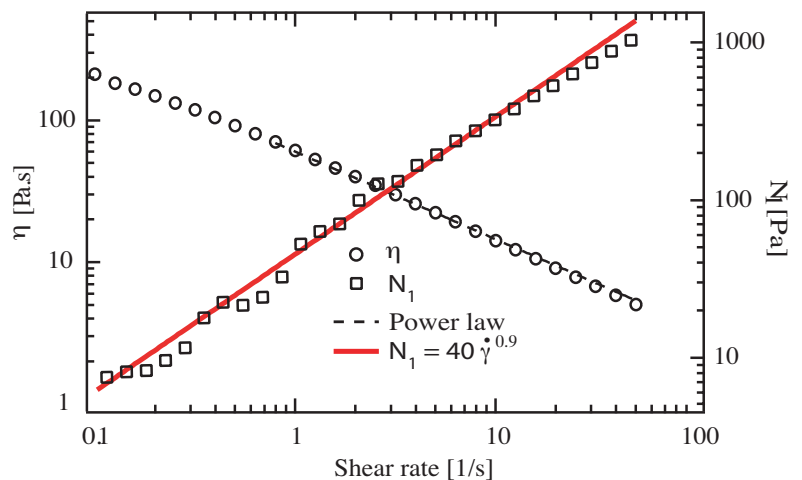

Figure 7. Rheological data for the test fluid containing $1.00 \mathrm{wt} \%$ PAA. (a) Storage $\left(G^{\prime}\right)$ and loss $\left(G^{\prime \prime}\right)$ moduli data as a function of frequency measured under small amplitude oscillatory conditions. The two lines indicated at low frequencies show the asymptotic variations $G^{\prime} \sim \omega, G^{\prime \prime} \sim \omega^{2}$ expected for simple Maxwell fluids in the limit of low frequency. (b) Viscosity (o) and first normal stress difference ( $\square$ ) data as a function of shear rate measured under steady shear conditions. The viscosity data at large shear rates is fitted using the power-law model $\eta=60|\dot{\gamma}|^{-0.55}$ (broken line), where $\dot{\gamma}$ has units of $\mathrm{s}^{-1}$. The first normal stress difference can be fitted by the function $\tau_{11}-\tau_{22}=40|\dot{\gamma}|^{0.90}$.

cus that forms close to the cylinder) to the lowest point of the cylinder. As the immersion depth varies, the local inclination angle $\alpha$ between the substrate and the horizontal at the point where the film is withdrawn from the fluid bath varies, resulting in different film thicknesses. The angle $\alpha$ is indicated in Figures 1 and 5. We took measurements at immersion depths $7 \mathrm{~cm}, 8 \mathrm{~cm}, 9 \mathrm{~cm}$ and $10 \mathrm{~cm}$ which correspond respectively to four different inclination angles, $\alpha=23^{\circ}, 47^{\circ}, 64^{\circ}$ and $79^{\circ}$.

The film thickness was measured using a digital micrometer located on a track that was securely attached to the roller apparatus. Before the roller started to rotate, we positioned the micrometer needle tip so that it just touched the cylinder surface and recorded that position as the zero point. When the dry cylinder was rotated through $2 \pi$ radians, the maximum deviation of the cylinder surface from the calibrated zero measurements was $12 \mu \mathrm{m}$. For each fluid at a given cylinder immersion depth, we slowly increased the rotation speed of the cylinder until a thin layer of fluid coated the roller uniformly. When a steady film was established, a film thickness measurement was made by reading the value shown when the micrometer needle tip just made direct contact with the surface of the fluid film. At a given rotation speed and inclination angle, five film thickness measurements were recorded at different 
locations along the axis, and we took the average value as the film thickness reading. The relative error of the film thickness measurement is around $8 \%$, and originates from the axisymmetric level of the cylinder and human error in the measurements. The rotation speed was increased in small increments and the flow was allowed to adjust to the new steady state before the next film thickness measurement was taken.

All rheological and experimental measurements were made at an ambient temperature of $25^{\circ} \mathrm{C}$. We ensured that reported results were reproducible by taking data multiple times and on multiple days. The fluids were thoroughly mixed prior to the roll coating experiments, and the measured change in the rheology of both fluids before and after the coating experiments was not significant. Finally, in order to verify the accuracy of the experimental setup and measurement technique, we compared the film thickness results we obtained using mineral oil (Aldrich) with the theoretically predicted film thickness $h_{\infty}^{N}$ in the Newtonian limit (Derjaguin, 1943; Landau and Levich, 1942; Wilson, 1981):

$$
h_{\infty}^{N}=1.34 \kappa_{m}^{-1}\left(\frac{\eta \Omega R}{\sigma}\right)^{2 / 3}=\frac{0.948(\eta \Omega R)^{2 / 3}}{[(1+\cos \alpha) \rho g]^{1 / 2} \sigma^{1 / 6}} .
$$

The mineral oil has constant viscosity $\eta=0.90$ Pa.s, surface tension $\sigma=0.060 \mathrm{~N} / \mathrm{m}$ and density $\rho=990 \mathrm{~kg} / \mathrm{m}^{3}$. Figure 8 shows that over a moderate range of rotation rates $\Omega$ the experimental data is in reasonable agreement with the theoretical prediction (34), represented by the solid and broken lines for the two different inclination angles $\alpha$. At a rotation rate of $1 \mathrm{rad} / \mathrm{s}$, the capillary number $\eta \Omega R / \sigma \approx 0.13$ and the relevant Reynolds number $\rho \Omega R h_{\infty}^{2} /(\eta \ell) \approx 0.6$; at a rotation rate of $20 \mathrm{rad} / \mathrm{s}$ the capillary and Reynolds numbers take the values 2.7 and 12 respectively. Therefore in this range inertial and gravitational effects start to become significant. It would appear that they have approximately equal and opposite effects, given the agreement with the theory that neglects both effects.

\section{Experimental results}

In this section we present the results of the experiments described in section 3 in which we measure the thickness of the film that coats a rotating roller as a function of rotation speed and of the depth of immersion of the cylinder. We consider the two cases in which the fluid rheology has weak (0.06 wt\% PAA) and strong (1.00 wt\% PAA) 


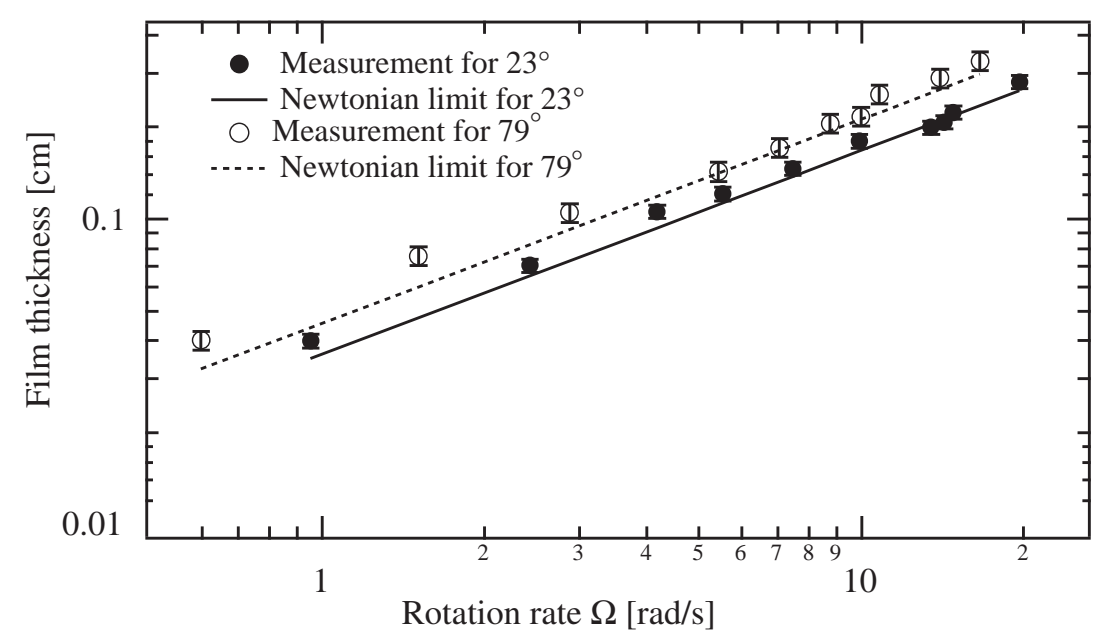

Figure 8. Experimental results for film thickness obtained with mineral oil as a function of the roller rotation rate $\Omega$, and the theoretically predicted film thickness (34) in the Newtonian limit. Measurements were made for two inclination angles, $\alpha=23^{\circ}$ and $79^{\circ}$.

viscoelastic properties, and compare our results with the theoretical predictions of film thickness derived in sections 2.4 and 2.5 respectively.

\subsection{Dilute Polymer solution (0.06 wT\% PAA)}

The measured rheology of the $0.06 \mathrm{wt} \%$ PAA solution was discussed in section 3, and the important rheological parameters are summarized in Table III. Viscoelastic effects are relatively weak in this fluid, and the most significant deviation from Newtonian behavior is the shearthinning observed in the viscosity.

We first consider the variations in film thickness with cylinder rotation rate. In figure 9a, the experimentally-measured film thickness is plotted as a function of the roller rotation rate when the inclination angle is $\alpha=47^{\circ}$. The error bars reflect the standard deviation of the five measurements made at different axial positions. Two theoretical predictions for film thickness are also plotted: firstly, the prediction (34) for a Newtonian liquid based on a viscosity equal to 0.067 Pa.s, which is the value of the zero shear rate viscosity of the $0.06 \mathrm{wt} \%$ fluid (broken line), and secondly prediction (28) for a fluid with weakly elastic rheology (solid line). The prefactor in (28) was calculated numerically to be $f(0.71)=0.90$ and therefore there are no free parameters in the weakly elastic prediction.

At sufficiently low rotation rates, the measured film thickness value is close to the prediction (34) based on Newtonian rheology. However, as the rotation rate increases above $\Omega \approx 2 \mathrm{rad} / \mathrm{s}$, shear-thinning effects 
in the fluid viscosity become significant and the film thickness decreases relative to the prediction for a Newtonian fluid. There is a transition region between rotation rates of 2 and $13 \mathrm{rad} / \mathrm{s}$ where the measured film thickness falls between the theoretical predictions in the Newtonian and weakly elastic limits, and then at higher shear rates the data are in good agreement with the predicted film thickness based on the weakly elastic theory. This is to be expected since the shear-thinning in the fluid viscosity is only significant above a certain shear-rate; below this shear rate, the viscosity is constant and is approximately equal to the zero shear rate viscosity (see Figure 6). The characteristic shear rate in the dynamic meniscus region can be simply estimated to be of order $\Omega R / h_{\infty}$. The experimental data shown in Figure 9 thus corresponds to shear rates in the range $280 \leq \dot{\gamma} \leq 1880 \mathrm{~s}^{-1}$. The rheological data in Figure 6 shows that the power-law model provides a good description of the rate-dependent viscosity over this range of shear rates. We attempted to capture the crossover from the power-law to the Newtonian limit quantitatively using theoretical methods by using a numerical program to solve the equation for film thickness based on the Ellis constitutive equation (Ashmore, 2003). However, we found that the crossover to the Newtonian limit was predicted to occur at significantly lower rotation rates than those observed in the experiments presented here.

Finally, when the rotation rate is higher than $\Omega_{c} \approx 26 \mathrm{rad} / \mathrm{s}$, the film thickness exceeds the value predicted based on the weakly elastic rheology, although it is still lower than the film thickness predicted for a Newtonian fluid. At rotation rates higher than those plotted on this graph, a hydrodynamic instability develops and the film becomes axially nonuniform. Overall, we find agreement between the experimental observations and the weakly elastic predictions in the range $13 \mathrm{rad} / \mathrm{s}$ $<\Omega<26 \mathrm{rad} / \mathrm{s}\left(1100 \mathrm{~s}^{-1}<\dot{\gamma}<1400 \mathrm{~s}^{-1}\right)$.

Next, we obtain a general result by nondimensionalizing the film measurements so that the rotation rate is converted to a capillary number. We also consider the variation of the film thickness with the depth of immersion of the roller. Motivated by the prediction based on weakly elastic theory, we plot a nondimensional film thickness $\mathcal{H}$, defined to be

$$
\mathcal{H}=\frac{h_{\infty}}{(1+\cos \alpha)^{3 /[2(2 n+1)]} R}=\left[9 f(n)^{3}\left(\frac{k(\Omega R)^{n}}{\sigma \kappa_{m}^{1-n}}\right)^{2}\right]^{1 /(2 n+1)} \frac{1}{\kappa_{m} R}(35)
$$

where the angular scaling is chosen such that according to the weakly elastic prediction (28), $\mathcal{H}$ is independent of the angle $\alpha$. Experimental plots of the function $\mathcal{H}$ are plotted as a function of the capillary number $k(\Omega R)^{n} /\left(\sigma \kappa_{m}^{1-n}\right)$ in figure $9 \mathrm{~b}$. Data for $\mathcal{H}$ based on four different inclination angles ranging from $23^{\circ}$ to $79^{\circ}$ is quantitatively similar when 

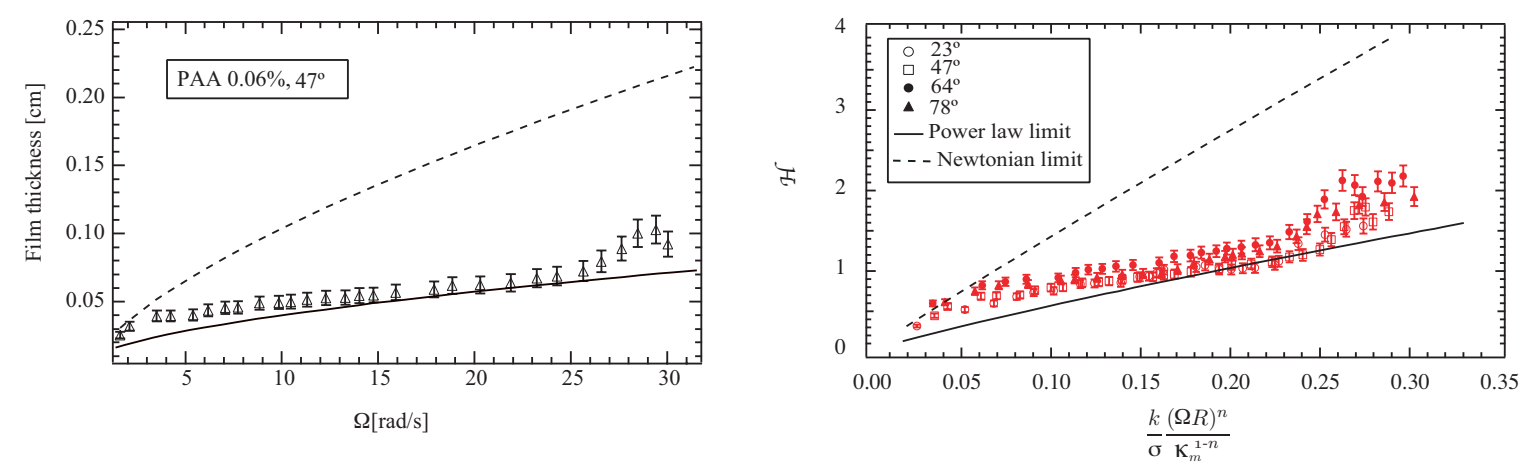

Figure 9. Experimental results for the film thickness generated using $0.06 \mathrm{wt} \% \mathrm{PAA}$, compared to theoretical predictions in both the Newtonian and weakly elastic limits. (a) Film thickness versus roller rotation rate at inclination angle $\alpha=47^{\circ}$. (b) Plot of dimensionless film thickness $\mathcal{H}$ defined in equation (35) versus $k(\Omega R)^{n} /\left(\sigma \kappa_{m}^{1-n}\right)$, where the values of the rheological parameters used are given in Table 2. Also marked are the theoretical predictions for the film thickness of a shear-thinning liquid (solid line) and a Newtonian liquid (dashed line).

$k(\Omega R)^{n} /\left(\sigma \kappa_{m}^{1-n}\right)<0.23$. At higher values, following the onset of the axial instability, the data departs from the weakly elastic prediction and the data shows increasing scatter owing to the spatial and temporal variations in the film thickness.

In the weakly elastic limit, the predicted and measured film thickness is considerably smaller than for a Newtonian fluid with (constant) viscosity equal to the zero shear rate viscosity. There is also a difference between the qualitative variation of the film thickness with rotation rate in the Newtonian and weakly elastic limits: in the Newtonian limit the film thickness varies as $\Omega^{2 / 3}$, whereas in the case of a weakly elastic shear-thinning liquid the thickness is proportional to $\Omega^{2 n /(2 n+1)}$.

The prediction of the weakly elastic model appears to agree with experimental data reasonably well when the nondimensional rotation rate $k(\Omega R)^{n} /\left(\sigma \kappa_{m}^{1-n}\right)$ falls between the values 0.13 and 0.23 . It should be noted that this apparently limited numerical range in fact corresponds to a factor of five variation in the rotation rate due to the powerlaw exponent of $2 n /(2 n+1) \approx 0.47$ in equation (35). Furthermore, the solid lines in Figures 9a,b are not a regression to the data but represent an a priori prediction of the dimensionless film thickness in terms of independently measured geometric and rheological properties.

\subsection{Semi-dilute polymer solution ( $1.00 \mathrm{Wt} \%$ PAA)}

The rheology of the $1.00 \mathrm{wt} \%$ PAA solution includes relatively strong viscoelastic effects, as shown by the measurements presented in sec- 
tion 3 and, in particular, the summary of the rheological data in Table III. Both the viscosity and the first normal stress difference exhibit shear-thinning over the measurable range of shear rates, and depend on the shear rate through a power-law relationship. We note that in the theoretical analysis presented in section 2, we assumed that the first normal stress coefficient varied as $\eta^{2}$, i.e. $\Psi_{1} \propto|\dot{\gamma}|^{2 n-2}$. The fits to the rheological data presented in section 3 resulted in the relationships $\eta \propto|\dot{\gamma}|^{-0.55}$ and $\Psi_{1}=\left(\tau_{11}-\tau_{22}\right) /|\dot{\gamma}|^{2} \propto|\dot{\gamma}|^{-1.1}$, which is consistent with both forms assumed in the theory when $n=0.45$.

In figure 10, we plot the measured film thickness as a function of rotation rate for four different inclination angles and compare the experimental data with the linear variation with $\Omega$ that is predicted in the strongly elastic limit (solid lines). Qualitatively, the variation does appear to be approximately linear, although some discrepancy is observed at low rotation rates. In addition, the angular dependence of the gradient $c(\alpha)$ obtained by fitting to the form $h_{\infty}=c(\alpha) \Omega$ is well represented by the variation $(1+\cos \alpha)^{-1 /(4 n)}$ predicted in the theoretical formula (32). Furthermore the deformation rates, $\dot{\gamma}=\Omega R / h_{\infty}$, spanned in these roll-coating experiments (corresponding to $40 \leq \dot{\gamma} \leq 120 \mathrm{~s}^{-1}$ ) is consistent with the range of shear rates over which power-law dependence of both the viscosity and the first normal stress difference can be observed in Figure 7. However, the value of the coefficient $g(n)$ which was calculated numerically proved to be significantly too large; we found $g(0.45)=0.48$, whereas the prefactor in the experimental data at $10 \mathrm{rad} / \mathrm{s}$ is on the order of 0.005 . We believe that the discrepancy between theory and experimental arises because one of the constraints on the theory is not satisfied in the experiments. This issue will be discussed further in section 5 .

We collapse the experimental data for different immersion depths by nondimensionalizing the film thickness using the following rescaling:

$$
\mathcal{H}=\frac{h_{\infty}}{R}\left(\frac{1+\cos \alpha}{2}\right)^{1 /(4 n)}=\left(\frac{N(\Omega R)^{2 n}}{\sigma}\right)^{1 /(2 n)}\left(\frac{\sigma}{\rho g}\right)^{1 /(4 n)},
$$

so that, using the prediction (32), the rescaled film thickness $\mathcal{H}$ is predicted to be independent of the inclination angle. The rescaled film thickness $\mathcal{H}$ is plotted as a function of the Deborah number $\mathcal{D}=\lambda \Omega$, which is the rotation rate nondimensionalized using the relaxation time $\lambda=3.3 \mathrm{~s}$, in Figure 11. The data are generally clustered close to the straight line. Therefore we conclude that the predicted qualitative variation of the film thickness with both the cylinder rotation rate and the inclination angle appears to be in good agreement with experimental data. 

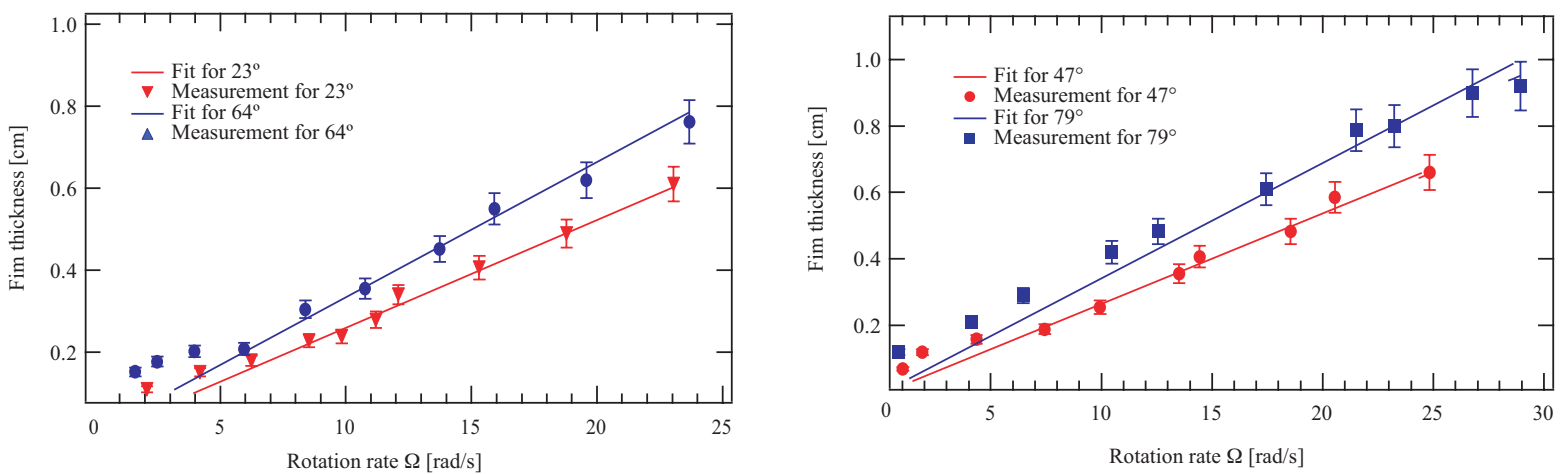

Figure 10. Film thickness versus roller rotation speed of $1.00 \mathrm{wt} \%$ PAA at four inclination angles: experimental data, with variations proportional to $\Omega R /(1+\cos \alpha)^{1 /(4 n)}$ also indicated (solid lines). This qualitative variation appears in the theoretical prediction (32) in the strongly elastic limit, although the prefactor calculated from the theory is significantly larger than the prefactor in the experimental data. The lines plotted are obtained by fitting the data to the functional form $c \Omega$, where $c\left(\alpha=23^{\circ}\right)=0.027, c\left(\alpha=47^{\circ}\right)=0.027, c\left(\alpha=62^{\circ}\right)=0.033, c\left(\alpha=79^{\circ}\right)=0.035$.

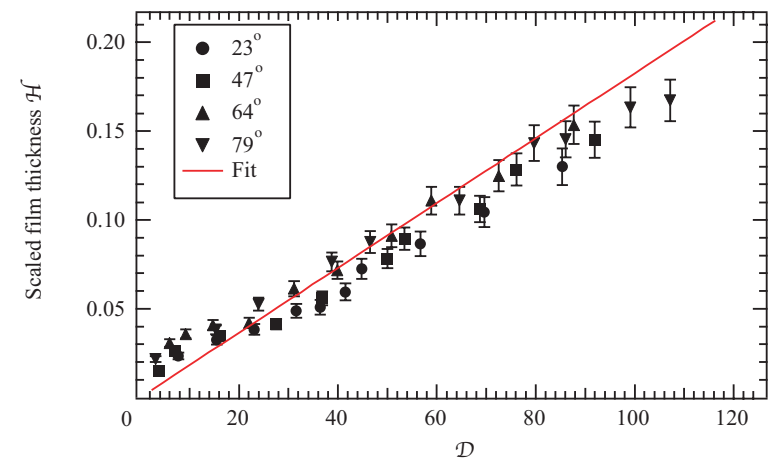

Figure 11. Dimensionless film thickness $\mathcal{H}$ defined in equation (36) versus Deborah number $\mathcal{D}=\lambda \Omega$ for a strongly elastic fluid $1.00 \mathrm{wt} \%$ PAA. The gradient of the straight line fitted to the data (solid line) is significantly smaller than the prefactor predicted by the theoretical formula (32).

\section{Discussion}

In the previous section we presented film thickness data for both $0.06 \mathrm{wt} \%$ and $1.00 \mathrm{wt} \%$ PAA solutions, and compared the results with theoretical predictions in the weakly and strongly elastic limits respectively. For the $0.06 \mathrm{wt} \%$ solution, we observed reasonable quantitative agreement with the theory in the weakly elastic limit over a range of rotation rates and for a number of different inclination angles. For the $1.00 \mathrm{wt} \%$ solution, the data showed the same trends as the theory for the strongly 
elastic limit as both rotation rate and inclination angle was varied, but the numerical prefactor predicted by the theory was substantially larger than that measured experimentally. In this section, we discuss this discrepancy as well as other aspects of the comparison between the data and the theory further. In particular, we discuss the values of the parameters $\tilde{\mathcal{C}}, \tilde{\mathcal{E}}, \mathcal{E}$ and the effective Reynolds number $\rho U^{2-n} h_{\infty}^{n+1} /(k \ell)$ for the $0.06 \mathrm{wt} \%$ and $1.00 \mathrm{wt} \%$ PAA solutions. We also present some data on the onset of an axial instability at high rotation rates.

The constraints on the values of $\tilde{\mathcal{C}}, \tilde{\mathcal{E}}, \mathcal{E}$ and $\rho U^{2-n} h_{\infty}^{n+1} /(k \ell)$ that apply to the theory in the weakly and strongly elastic limits are summarized in Table II and Figure 4. We first consider the values of $\tilde{\mathcal{C}}$ and $\mathcal{E}$ for the $0.06 \mathrm{wt} \%$ solution and of $(\tilde{\mathcal{C}} \tilde{\mathcal{E}})^{1 /(4 n)}$ and $\mathcal{E}$ for the $1.00 \mathrm{wt} \%$ solution, and we tabulate the calculated values for both fluids over a range of rotation rates in Table IV. For the $0.06 \mathrm{wt} \% \mathrm{PAA}$, the capillary number $\tilde{\mathcal{C}}$ is less than unity at all values of the rotation rate, as required for the theory in the weakly elastic limit to be valid. The elasticity parameter $\mathcal{E}$ does take values greater than unity, although strictly speaking the weakly elastic theory is valid for $\mathcal{E} \ll 1$.

Also, the theory neglected inertial effects, which corresponds to the condition that

$$
\frac{\rho U^{2-n} h_{\infty}^{n+1}}{k \ell}=\frac{\rho(\Omega R)^{2} \kappa_{m}^{-1}}{\sigma} \ll 1
$$

in the weakly elastic limit. Note that with the chosen scaling the effective lubrication Reynolds number in (37), characterizing the magnitude of inertial stresses relative to viscous stresses in this flow, is a Weber number. As can be seen from the values tabulated in Table IV, in the experiments this parameter exceeds the value unity when the rotation rate is greater than $5 \mathrm{rad} / \mathrm{s}$, and it is therefore surprising that we obtain quantitative agreement between the experimental data and the theory, which neglects inertial effects. We investigated the consequences of inertial effects for the $0.06 \mathrm{wt} \%$ PAA solution both theoretically and experimentally, and a more detailed theoretical analysis of the magnitude of inertial effects in the weakly elastic limit is presented in Appendix B. A comparison between the analysis of Appendix B and the experimental results implies that the nondimensional constant that characterizes the strength of inertial effects, which varies with the power-law index $n$, is smaller when the fluid is shear-thinning $(n<1)$ than in the Newtonian limit $(n=1)$.

For the $1.00 \mathrm{wt} \% \mathrm{PAA}$, the value of the parameter $\mathcal{E}$ always exceeds unity as is required for the strongly elastic theory to be valid. However, the product of the modified capillary and elasticity numbers $\tilde{\mathcal{C}} \tilde{\mathcal{E}}$ is not small compared to unity which violates the second condition that must 
be satisfied for the theory to be valid. In addition, inertial (including centrifugal) effects are neglected in the theory which leads to the following constraint

$$
\frac{\rho U^{2-n} h_{\infty}^{n+1}}{k \ell}=\frac{\rho}{k(\Omega R)^{n-2} \kappa_{m}^{n}}\left(\frac{N(\Omega R)^{2 n} \kappa_{m}^{2 n-1}}{\sigma}\right)^{(2 n+1) /(4 n)} \ll 1
$$

in the strongly elastic limit. In the experiments on the $1.00 \mathrm{wt} \%$ PAA solution, this parameter is significantly larger than unity when the rotation rate exceeds $5 \mathrm{rad} / \mathrm{s}$. Therefore two constraints on the theory are violated, and as a result the modeled velocity field and resulting thin film equation may not be an entirely accurate characterization of the physical system. This is the most likely explanation for the discrepancy between the theoretical predictions and experimental measurements. In general, the experimental trend would be qualitatively different from the theory, but we observe only a difference in the quantitative prefactors.

This leads us to a comment about the significance of the substrate geometry in the non-Newtonian coating problem. In Newtonian coating flows the curvature of the static meniscus, which depends on the geometry of the coating substrate, does not enter the problem when the length-scales are nondimensionalized by $\kappa_{m}$. Therefore the range of the validity of the low capillary number result only depends on the value of the capillary number in the Newtonian limit $\mathcal{C}_{N}=\eta U / \sigma$ and is independent of the curvature. The substrate geometry thus plays a limited role in the Newtonian problem. In contrast, in the nonNewtonian coating problem we analyze in this paper, the curvature of the meniscus $\kappa_{m}$ affects the values of $\tilde{\mathcal{C}}$ and $\tilde{\mathcal{E}}$. When other parameters are held constant, a larger value of the curvature (i.e. smaller radius of curvature) yields a smaller value of $\tilde{\mathcal{C}}$ and a larger value of $\tilde{\mathcal{E}}$. Also, the importance of inertial effects decreases as the curvature increases. Therefore the conditions in the theory that are violated in the present experiments using a roller with diameter $8.3 \mathrm{~cm}$ are more likely to be satisfied in experiments using a thin fiber (de Ryck and Quéré, 1998).

Finally, we comment briefly on the onset of an axial instability that leads to periodic variations in the film thickness. A representative image of the ribbing instability observed in the weakly elastic case is shown in Figure 12. In this image (viewed from the downstream side, i.e. an azimuthal angle of $\theta \approx 3 \pi / 2$ ) the top of the cylinder is rotating towards the reader. The free surface shows periodic ribs along the axial length of the cylinder. The ribs have an approximately constant wavelength but have a complicated axial structure. Values of the critical rotation rate and the critical film thickness at which the instability develops are 
Table IV. Values of the dimensionless parameters $\tilde{\mathcal{C}}, \mathcal{E}$ and $\rho U^{2-n} h_{\infty}^{n+1} /(k \ell)$ for both the $0.06 \mathrm{wt} \%$ PAA and $1.00 \mathrm{wt} \%$ PAA for selected values of the cylinder rotation rate. The expression for $\rho U^{2-n} h_{\infty}^{n+1} /(k \ell)$ for the $0.06 \mathrm{wt} \%$ PAA fluid is given in terms of the material parameters by (37), and the expression for $1.00 \mathrm{wt} \% \mathrm{PAA}$ is given by (38). We take $\alpha=0$ in calculating the value of the curvature $\kappa_{m}$, and use the estimate of the first normal stress coefficient in the limit of zero shear-rate for the value of $N$ for $0.06 \mathrm{wt} \%$ PAA. The theoretical constraints on the parameters tabulated here are summarized in Table II and Figure 4.

\begin{tabular}{llllll}
\hline & & \multicolumn{4}{c}{ rotation rate $[\mathrm{rad} / \mathrm{s}]$} \\
& wt\% PAA & 1 & 5 & 10 & 25 \\
\hline$\tilde{\mathcal{C}}=3 k U^{n} /\left(\sigma \kappa_{m}^{1-n}\right)$ & 0.06 & $8.7 \times 10^{-4}$ & $2.7 \times 10^{-3}$ & $4.5 \times 10^{-3}$ & $8.6 \times 10^{-3}$ \\
\hline$(\tilde{\mathcal{C}} \tilde{\mathcal{E}})^{1 /(4 n)}=3 N U^{2 n} /\left(\sigma \kappa_{m}^{1-2 n}\right)$ & 1.00 & 10 & 23 & 32 & 51 \\
\hline $\mathcal{E}=\tilde{\mathcal{E}} / \tilde{\mathcal{C}}^{(2 n-1) /(2 n+1)}$ & 0.06 & 0.7 & 1.8 & 2.7 & 4.6 \\
$=\left[\tilde{\mathcal{E}}^{2 n+1} / \tilde{\mathcal{C}}^{2 n-1}\right]^{1 /(4 n)}$ & 1.00 & 2.4 & 4.6 & 6.0 & 8.6 \\
\hline$\rho U^{2-n} h_{\infty}^{n+1} /(k \ell)$ & 0.06 & 0.073 & 1.8 & 7.3 & 46 \\
& 1.00 & 0.23 & 13 & 71 & 700 \\
\hline
\end{tabular}

tabulated in Table $\mathrm{V}$ for the mineral oil, and the $0.06 \mathrm{wt} \%$ and $1.00 \mathrm{wt} \%$ PAA solutions. Such instabilities can arise from either inertial effects (Moffatt, 1977) or elastic effects, resulting from the interaction of the first normal stress with the streamline curvature (Joo and Shaqfeh, 1992; Graham, 2003). Although analysis of this instability is not the primary focus of the present work a number of important factors can be discerned from this data and previous theoretical studies in related geometries. Firstly, we note that purely inertial instabilities (e.g. Moffatt, 1977) and purely elastic-driven instabilities (e.g. Grillet et al., 1999; Joo and Shaqfeh, 1992; Graham, 2003) have been studied in single roll coating applications. Furthermore detailed studies in the Taylor-Couette geometry have shown that in addition to purely elastic instabilities arising from streamline curvature (Larson et al., 1990; Muller et al., 1989), weak elastic effects may stabilize or destabilize inertially-driven flow transitions. These competing effects can be represented by stability loci plotted on a diagram of the critical Deborah number versus critical Reynolds number. The data in Table $\mathrm{V}$ indicate that weak elastic effects (i.e. shear thinning in the fluid viscosity) significantly reduce the critical film thickness at onset of instability but in general shift the critical rotation rate to higher values. In contrast strong elastic effects in the $1.00 \mathrm{wt} \%$ PAA solution lead to thicker films at the onset of instability but substantially lower critical rotation rates. Lopez et al. 
Table V. The onset of instability: table showing the critical rotation rate and critical film thickness for the mineral oil and the $0.06 \mathrm{wt} \%$ PAA and $1.00 \mathrm{wt} \%$ PAA solutions.

\begin{tabular}{llllll}
\hline & angle & $23^{\circ}$ & $47^{\circ}$ & $64^{\circ}$ & $79^{\circ}$ \\
\hline critical rotation rate & mineral oil & 15 & 21 & 20 & 17 \\
$\Omega_{\text {crit }}[\mathrm{rad} / \mathrm{s}]$ & $0.06 \mathrm{wt} \%$ PAA & 21 & 22 & 15 & 18 \\
& $1.00 \mathrm{wt} \%$ PAA & 8.5 & 9.9 & 8.4 & 17 \\
\hline critical film thickness & mineral oil & 0.22 & 0.32 & 0.33 & 0.33 \\
$h_{\text {crit }}[\mathrm{cm}]$ & $0.06 \mathrm{wt} \%$ PAA & 0.059 & 0.064 & 0.062 & 0.07 \\
& $1.00 \mathrm{wt} \%$ PAA & 0.32 & 0.35 & 0.33 & 0.46 \\
\hline
\end{tabular}

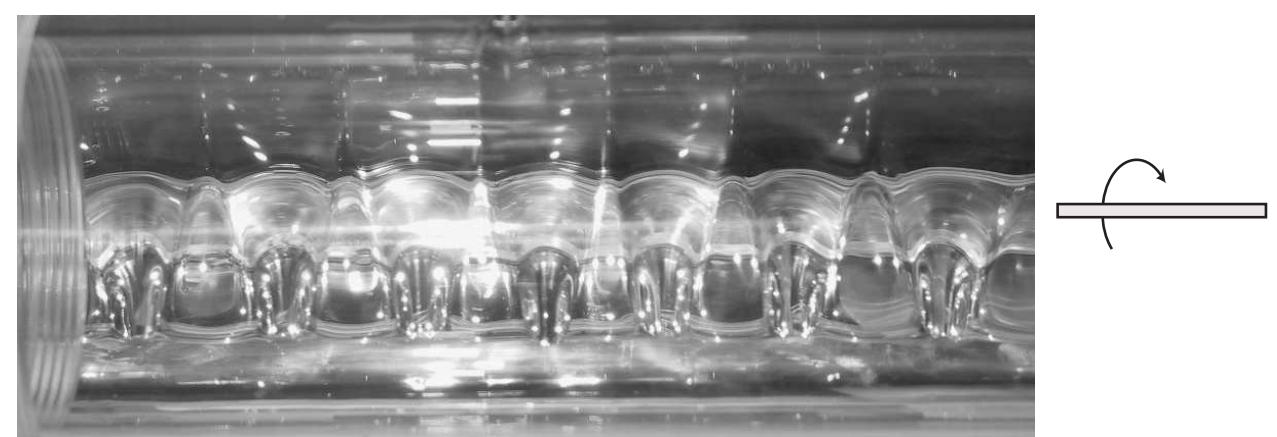

Figure 12. Photograph of axial instability observed for $0.06 \mathrm{wt} \%$ PAA with rotation rate $41.2 \mathrm{rad} / \mathrm{s}\left(\Omega / \Omega_{\text {crit }}=1.88\right)$ and inclination angle $47^{\circ}$. The wavelength is approximately $L_{Z}=2.5 \mathrm{~cm}\left(L_{Z} / R=0.60\right)$.

(2002) examined non-Newtonian effects on the transition to a ribbing instability, the case in which one film splits into two in a diverging gap, and found a weak reduction in the critical rotation rate at low polymer concentrations, but a qualitatively similar strong reduction in the critical rotation rate in elastic fluids with high polymer concentrations.

\section{Conclusions}

In this paper we have presented a theoretical analysis of the film thickness that is generated by coating flows in a variety of geometries (a plate, a roller, a fiber and inside a cylindrical tube) based on the CEF constitutive equation. We have focused on the low Reynolds number regime, and have analyzed two different limits: in the weakly elastic limit, shear-thinning of the viscosity dominates and the elastic stress 
is unimportant. The film thickness is determined by a balance between viscous and surface tension forces, as in the Newtonian limit, although the shear-rate dependence of the viscosity significantly reduces the resulting film thickness. In the strongly elastic limit, the effects of the elastic stress dominate and compete against surface tension to set the film thickness. The effect of viscosity, although not entirely negligible, is relatively weak in this limit. The expression for the film thickness in terms of the variables in the problem in the two different limits treated here, and the conditions for validity, are summarized in Table II. The theory predicts that in the weakly elastic limit the film thickness is less than in the Newtonian limit, whereas in the strongly elastic limit it is greater. The theoretical results presented in this paper represent the first detailed analysis of film coating with fluids characterized by the CEF constitutive equation, and provide a more general description of the physical processes that set the film thickness than other works which have focused on a single geometry (with the exception of the work of Ro and Homsy (1995) who presented an analysis on the basis of the Oldroyd-B constitutive equation). The analytical results are summarized in Table II and Figure 4.

We have also performed experiments in the single roller geometry and have compared the experimentally-observed values of the film thickness with our predictions for two different well-characterized fluids, one weakly elastic and the other strongly elastic. Our results generally show good qualitative agreement, with small deviation, at low rotation rates. In the weakly elastic limit, the quantitative agreement is good also, which is notable since earlier studies with less fully characterized fluids have often found significant discrepancies (e.g. Gutfinger and Tallmadge, 1965; Spiers et al., 1974). The lack of quantitative agreement between the prediction of the strongly elastic theory and the experimental measurements is most likely to be explained by the modified capillary and Reynolds numbers being relatively large in the experiments. Furthermore as we noted in section 2.3, the effective Deborah number, measuring the relative importance of streamwise extension (i.e. non-viscometric kinematics of the flow) may also become significant for large rotation rates. The CEF model cannot adequately capture such phenomena and it becomes necessary to pursue large-scale freesurface numerical computations of the roll-coating problem using an appropriate viscoelastic constitutive equation such as the Oldroyd-B model. The ability to perform convergent and stable computations of such problems with viscoelastic fluids is presently under development (Pasquali and Scriven, 2002; Bhatara et al., 2004). The analytic results developed in the present paper provide rigorous asymptotic limits against which the predictions of such codes may be compared. 


\section{Acknowledgements}

We are grateful to Dr. J.M. Rallison for pointing out the contribution of viscoelastic terms to the shear stress and to Professor M.M. Denn for drawing our attention to the validity of the CEF model being restricted to small Deborah number flows. We also benefitted from numerous useful discussions with, and financial support from, colleagues at PPG Industries Inc.

\section{Appendix A: A more exact film thickness prediction in the weakly elastic limit}

As noted in section 2.2, the only means of deriving an equation for $h\left(x_{1}\right)$ when using the full CEF constitutive equation and including the effects of a first normal stress difference was to assume an approximate form of the velocity field. In this section, we compare the solution (28) based on the approximate velocity field (18), which accounts for the effects of elastic stress (although these are neglected later in the analysis), with a solution based on a more accurate velocity field which does not account for elastic stress effects. The analysis discussed in this section corresponds to using the power-law constitutive equation, which was described for the geometry of a flat plate and compared to experimental results by Gutfinger and Tallmadge (1965); here we consider a more general coating geometry.

If the normal stress differences are neglected and a power-law form is used for the viscosity $\eta=k|\dot{\gamma}|^{n-1}$, equation (16) becomes

$$
\mathcal{C}^{-1} \frac{d \kappa}{d x_{1}}-\frac{\partial}{\partial x_{2}}\left(-\frac{\partial u_{1}}{\partial x_{2}}\right)^{n}-\mathcal{G} \sin \alpha=0
$$

and the exact form of the velocity field is

$u_{1}\left(x_{1}, x_{2}\right)=1+\frac{n}{n+1}\left(\mathcal{G} \sin \alpha-\mathcal{C}^{-1} \frac{d \kappa}{d x_{1}}\right)^{1 / n}\left[\left(h-x_{2}\right)^{1+1 / n}-h^{1+1 / n}\right]$.

Integrating to obtain the flux equation, we find that

$$
1=h-\frac{n}{(2 n+1)}\left(\mathcal{G} \sin \alpha-\mathcal{C}^{-1} \frac{d \kappa}{d x_{1}}\right)^{1 / n} h^{2+1 / n},
$$

which is very similar to a rearrangement of the equation (23) obtained using the full $\mathrm{CEF}$ model $(22)$ in the limit $\mathcal{E} \ll 1$ :

$$
1=h-\frac{1}{3}\left(\mathcal{G} \sin \alpha-\mathcal{C}^{-1} \frac{d \kappa}{d x_{1}}\right)^{1 / n} h^{2+1 / n} .
$$


The prefactor $1 / 3$ in the general CEF derivation has been replaced by $n /(2 n+1)$ in the power-law derivation. The effect on the final result for the film thickness can be represented by replacing $\tilde{\mathcal{C}}$ in $(28)$ by the following capillary number:

$$
\tilde{\mathcal{C}}_{P L}=\left(\frac{2 n+1}{n}\right)^{n} \frac{k U^{n} \kappa_{m}^{n-1}}{\sigma} .
$$

The value of $f(n)$ is identical in the more precise calculation. This result shows that the approximation made in the derivation for the general CEF model, namely the assumption of quadratic variation in the velocity field, only affects the prefactor in the film thickness prediction, not the dynamic scaling. The error increases as $n$ deviates from the value of unity.

\section{Appendix B: Weak inertial effects in non-Newtonian coating}

The analysis leading to equation (22) for the film thickness assumes that inertial effects are negligible and therefore is only valid when the effective Reynolds number $\left(\rho U h_{\infty} / \eta\right)\left(h_{\infty} / \ell\right) \ll 1$, as indicated in Table II. In this Appendix, we extend this analysis to account for the effect of weak inertial forces on a thin liquid film with rheology consistent with the weakly elastic limit and discuss our experimental measurements in light of this result. A similar analysis could also be performed for a strongly elastic fluid.

When the fluid is Newtonian, substituting the expressions for the film thickness $h_{\infty}$ and the length-scale $\ell$ into the effective Reynolds number $h_{\infty} \mathcal{R} / \ell$ yields the Weber number $\mathcal{W}=\rho U^{2} \kappa_{m}^{-1} / \sigma$. In the limit that inertial effects are weak, i.e. $\mathcal{W}<1$, Quéré and de Ryck (1996) used a scaling analysis to quantify the effect of inertial forces on the predicted film thickness (34), and found that

$$
h_{\infty}=\frac{1.34}{\left(1-c_{1}^{N} \mathcal{W}\right)}\left(\frac{\eta U}{\sigma}\right)^{2 / 3} \kappa_{m}^{-1},
$$

where $c_{1}^{N}$ is a constant that must be determined. The experimental results of Quéré and de Ryck (1996) show that the film thickness of a Newtonian fluid does increase due to inertial effects when $\mathcal{W}<1$, in qualitative agreement with the prediction (44) and in agreement with physical intuition. The value of the constant $c_{1}^{N}$ was calculated from experimental results by Shen et al. (2002), and was found to take the value 0.65 for water and 0.69 for 35 wt $\%$ glycerin with water. The 
scaling analysis that leads to equation (44) is likely to break down when $\mathcal{W}>1$, and therefore the divergence in the film thickness that occurs when $\mathcal{W}=1 / c_{1}^{N}>1$ is outside the range of validity of the equation.

When the fluid is weakly elastic, the scalings of the inertial, capillary pressure and viscous stress gradients in the flow $\left(x_{1}\right)$ direction are

$$
-\frac{\rho U^{2}}{\ell} \approx-\frac{\sigma \kappa_{m}}{\ell}+\frac{k U^{n}}{h_{\infty}^{n+1}}
$$

respectively. Signs have been chosen appropriately, based on a detailed understanding of the velocity and interfacial gradients. Also, the scaling that represents the forces in the meniscus is

$$
\kappa_{m}-\frac{h_{\infty}}{\ell^{2}} \approx \frac{\rho U^{2}}{\sigma} .
$$

This result is the analog of the curvature matching requirement (see e.g. (27)), accounting for inertial effects. Eliminating $\ell$ from (45) and (46), and introducing unknown constants $c_{1}(n), c_{2}(n)$ since we have so far only used scaling estimates to approximate the size of terms, leads to

$$
h_{\infty}=\frac{c_{2}(n)}{\left(1-c_{1}(n) \mathcal{W}\right)^{3 /(2 n+1)}}\left(\frac{k U^{n}}{\sigma \kappa_{m}^{1-n}}\right)^{2 /(2 n+1)} \kappa_{m}^{-1} .
$$

Using knowledge of the prefactor when $\mathcal{W} \ll 1$ (see equation (28)) enables us to write

$$
h_{\infty}=\left[9\left(\frac{f(n)}{1-c_{1}(n) \mathcal{W}}\right)^{3}\left(\frac{k U^{n}}{\sigma \kappa_{m}^{1-n}}\right)^{2}\right]^{1 /(2 n+1)} \kappa_{m}^{-1} .
$$

If the value of the constant $c_{1}(0.71)$ were close to the value determined in the Newtonian limit, $c_{1}(1)=c_{1}^{N} \approx 0.7$, we would expect to see significant deviation between the experimental results for the $0.06 \mathrm{wt} \%$ solution and the theoretical prediction (28). Since this is not the case, we deduce that the value of $c_{1}(0.71)$ is likely to be significantly smaller than 0.7 .

Additional experiments were performed so that the Weber number $\mathcal{W}=\rho(\Omega R)^{2} \kappa_{m}^{-1} / \sigma$ was reduced, i.e. inertial effects were weaker, relative to the value in the measurements presented in section 4 , and another set of film thickness data was taken. The fluid used was the same $0.06 \mathrm{wt} \%$ PAA solution but, since the experiments were performed sometime after the solution was first mixed, the solution had aged and the rheology had changed slightly. We distinguish between the different rheologies by referring to the first batch of results, presented 
in section 4, as results from solution $\mathrm{A}$ and the results we present in this section as results from solution B. Solution B had a density of $1080 \mathrm{~kg} / \mathrm{m}^{3}$ and a surface tension of $55 \times 10^{-3} \mathrm{~N} / \mathrm{m}$. The power-law variation in the viscosity was fitted using the curve $\eta(\dot{\gamma})=0.3|\dot{\gamma}|^{-0.54}$, i.e. $n=0.46$. Whereas the Weber number in the experiments on solution A ranged from 0.089 upwards, in the experiments on solution B the Weber number fell in the range 0.012 to 1.7. In the experiments on solution $\mathrm{B}$, the angle $\alpha=50^{\circ}$. The film thickness was measured using a laser confocal surface metrology system (LT-8110, Keyence Inc.), rather than the direct method described in section 3. A detailed description of this measurement method is given by Kavehpour et al. (2002). The measurements had a vertical resolution of $0.5 \mu \mathrm{m}$ and the film thickness was measured at the top of the cylinder. The results for solutions A and $\mathrm{B}$ are plotted in Figure 13, and are compared against the theoretical prediction (28).

Although the film thickness measurements for solution B are made under conditions which correspond to small Weber number and small capillary number, the experimental data give a film thickness approximately twice as large as the theoretically predicted value. As can be observed from the plot, this data is in the range of rotation rates where discrepancies were also observed between experimental measurements and theoretical predictions for solution A. The discrepancy between the experimental data and theoretical prediction is of a similar magnitude for both solutions A and B despite the difference in the Weber numbers. Therefore it would appear that inertia has only a small effect, even when the Weber number is of order unity.

\section{References}

Ashmore, J. Free-surface and coating flows, Ph.D. Thesis, Harvard University, 2003. Bird, R.B., Armstrong, R.C. and Hassager, O. Dynamics of Polymeric Liquids, Volume 1, Wiley Interscience 1987.

Bhatara, G., Shaqfeh, E.S.G. and Khomami, B. Influence of visco-elasticity on the interfacial dynamics of air displacing fluid flows - A computational study $J$. Non-Newtonian Fluid Mech. (to appear), 2004.

Barnes, H.A., Hutton, J.F. and Walters, K. An Introduction to Rheology, Elsevier, Amsterdam, 1989.

Bretherton, F.P. The motion of long bubbles in tubes J. Fluid Mech. 10, 166-188, 1961.

Campanella, O.H. and Cerro, R.L. Viscous flow on the outside of a horizontal rotating cylinder: the roll coating regime with a single fluid Chem. Eng. Sci. 39, 1443-1449, 1982.

Campanella, O.H., Galazzo, J.L. and Cerro, R.L. Viscous flow on the outside of a horizontal rotating cylinder-II. Dip coating with a non-Newtonian fluid Chem. Eng. Sci. 41, 2707-2713, 1982. 


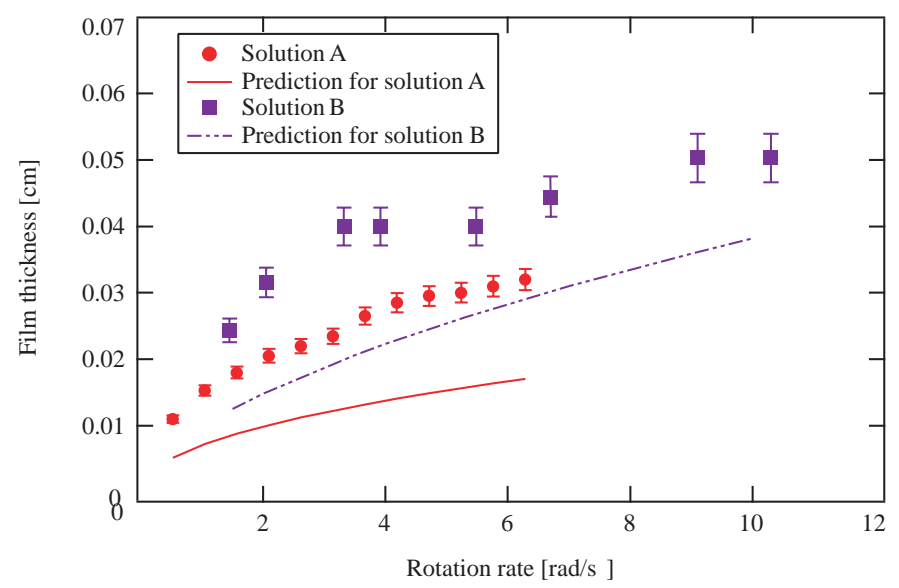

Figure 13. Measured and theoretically predicted film thickness versus rotation rate for solution A and solution B, both made with $0.06 \mathrm{wt} \%$ PAA. The solution A results are a subset of those presented in Figure 9a.

Cerro, R.L. and Scriven, L.E. Rapid free surface film flows. An integral approach Ind. Eng. Chem. Fundam. 19, 40-50, 1980.

Derjaguin, B. Thickness of liquid layer adhering to walls of vessels on their emptying and the theory of photo- and motion picture film coating Comptes Rendus (Doklady) de l'Academie des Sciences de l'URSS XXXIX, 13-16, 1943.

Graham M.D. Interfacial hoop stress and instability of viscoelastic free surface flows Phys. Fluids 15, 1702-1710, 2003.

Grillet, A.M., Lee, A.G. and Shaqfeh, E.S.G. Observations of ribbing instabilities in elastic flows with gravity stabilization J. Fluid Mech. 399, 49-83, 1999.

Gutfinger, C. and Tallmadge, J.A. Films of non-Newtonian fluids adhering to flat plates A.I.Ch.E. Journal 11, 403-413, 1965.

Huilgol R.R. On the concept of the Deborah number Trans. of the Soc. of Rheol. 19, 297, 1975.

Joo, Y.L. and Shaqfeh, E.S.G. The effects of inertia on the viscoelastic Dean and Taylor-Couette flow instabilities with application to coating flows Phys. Fluids A 4, 2415-2431, 1992.

Kavehpour, P., Ovryn, B. and McKinley, G.H. Evaporatively-driven Maragoni instabilities of volatile liquid films spreading on thermally-conductive substrates Colloids and Surf. A 206, 409-423, 2002.

Kistler, S.F. and Scriven, L.E. Coating flows in Computational Analysis of Polymers, ed. J. Pearson, S. Richardson, Chap. 8. Barking, Essex, Engl: Appl. Sci. 343, 1983.

Kizito, J.P., Kamotani, Y. and Ostrach, S. Experimental free coating flows at high capillary number Experiments in Fluids 27, 235-243, 1999.

Kulicke, W.-M. and Clasen, C. Viscosimetry of Polymers and Polyelectrolytes Springer, 2004.

Landau, L. and Levich, B. Dragging of liquid by a plate Acta Physiochim. USSR 17, 42-54, 1942.

Larson, R.G., Shaqfeh, E.S.G. and Muller, S.J. A purely elastic instability in TaylorCouette flow J. Fluid Mech 218, 573-600, 1990. 
Lopez, F.V., Pauchard, L., Rosen, M. and Rabaud, M. Non-Newtonian effects on ribbing stability threshold J. Non-Newton. Fluid Mech. 103 (2-3), 123-139, 2002 .

Middleman, S. Free coating of viscous and viscoelastic liquids onto a partially submerged rotating roll Polymer Eng. and Sci. 18, 734-737, 1978.

Moffatt, H.K. Behaviour of a viscous film on the outer surface of a rotating cylinder J. de Mecanique 16, 651-673, 1977.

Muller, S.J., Larson, R.G. and Sahqfeh, E.S.G. A purely elastic transition in TaylorCouette flow Rheol. Acta 28, 499-503, 1989.

Pasquali, M. and Scriven, L.E. Free surface flows of polymer solutions with models based on the conformation tensor J. Non-Newtonian Fluid Mech. 108, 363-409, 2002.

Quéré, D. and de Ryck, A. Inertial coating of a fiber J. Fluid Mech. 311, 219-237, 1996.

Quéré D. Fluid coating on a fiber Ann. Rev. Fluid Mech. 31, 347-384, 1999.

de Ryck, A. and Quéré, D. Fluid coating from a polymer solution Langmuir 14, 1911-1914, 1998.

Ro, J.S. and Homsy, G.M. Viscoelastic free surface flows: thin film hydrodynamics of Hele-Shaw and dip coating flows J. Non-Newton. Fluid Mech. 57, 203-225, 1995.

Ruschak, K.J. Coating flows Ann. Rev. Fluid Mech. 17, 65-89, 1985.

Shen, A.Q., Gleason, B., McKinley, G.H. and Stone, H.A. Fiber coating with surfactant solutions Phys. Fluids 14 (11), 4055-4068, 2002.

Spiers, R.P., Subbaraman, C.V. and Wilkinson, W.L. Free coating of a Newtonian liquid onto a vertical surface Chem. Eng. Sci. 29, 389-396, 1974.

Spiers, R.P., Subbaraman, C.V. and Wilkinson, W.L. Free coating of non-Newtonian liquids onto a vertical surface Chem. Eng. Sci. 30, 379-395, 1975.

Tallmadge, J.A. A withdrawal theory for Ellis model fluids A.I.Ch.E. Journal 12, 1011-1014, 1966.

Tharmalingham, S. and Wilkinson, W.L. The coating of Newtonian liquids onto a roll rotating at low speeds Polymer Eng. and Sci. 18, 1155-1159, 1978.

Wilson, S.D.R. The drag-out problem in film coating theory J. Eng. Math. 16, 209-221,1981. 